\title{
Targeting the CXCR4 pathway using a novel anti-CXCR4 IgG1 antibody (PF- 06747143) in chronic lymphocytic leukemia
}

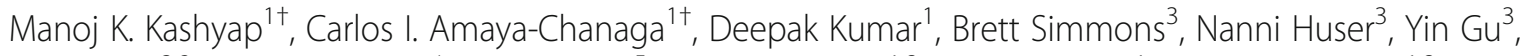
Max Hallin ${ }^{3,8}$, Kevin Lindquist ${ }^{4}$, Rolla Yafawi ${ }^{5}$, Michael Y. Choi ${ }^{1,2}$, Ale-Ali Amine ${ }^{1}$, Laura Z. Rassenti ${ }^{1,2}$, Cathy Zhang ${ }^{3}$, Shu-Hui Liu ${ }^{4}$, Tod Smeal ${ }^{3,6}$, Valeria R. Fantin ${ }^{3,7}$, Thomas J. Kipps ${ }^{1,2}$, Flavia Pernasetti ${ }^{3^{*}}$ (DD and Januario E. Castro ${ }^{1,2^{*}}$

\begin{abstract}
Background: The CXCR4-CXCL12 axis plays an important role in the chronic lymphocytic leukemia (CLL)-microenvironment interaction. Overexpression of CXCR4 has been reported in different hematological malignancies including CLL. Binding of the pro-survival chemokine CXCL12 with its cognate receptor CXCR4 induces cell migration. CXCL12/CXCR4 signaling axis promotes cell survival and proliferation and may contribute to the tropism of leukemia cells towards lymphoid tissues and bone marrow. Therefore, we hypothesized that targeting CXCR4 with an IgG1 antibody, PF-06747143, may constitute an effective therapeutic approach for CLL.

Methods: Patient-derived primary CLL-B cells were assessed for cytotoxicity in an in vitro model of CLL microenvironment. PF-06747143 was analyzed for cell death induction and for its potential to interfere with the chemokine CXCL12-induced mechanisms, including migration and F-actin polymerization. PF-06747143 in vivo efficacy was determined in a CLL murine xenograft tumor model.

Results: PF-06747143, a novel-humanized IgG1 CXCR4 antagonist antibody, induced cell death of patient-derived primary CLL-B cells, in presence or absence of stromal cells. Moreover, cell death induction by the antibody was independent of CLL high-risk prognostic markers. The cell death mechanism was dependent on CXCR4 expression, required antibody bivalency, involved reactive oxygen species production, and did not require caspase activation, all characteristics reminiscent of programmed cell death (PCD). PF-06747143 also induced potent B-CLL cytotoxicity via Fc-driven antibody-dependent cell-mediated cytotoxicity (ADCC) and complement-dependent cytotoxicity activity (CDC). PF-06747143 had significant combinatorial effect with standard of care (SOC) agents in B-CLL treatment, including rituximab, fludarabine (F-ara-A), ibrutinib, and bendamustine. In a CLL xenograft model, PF-06747143 decreased tumor burden and improved survival as a monotherapy, and in combination with bendamustine.
\end{abstract}

Conclusions: We show evidence that PF-06747143 has biological activity in CLL primary cells, supporting a rationale for evaluation of PF-06747143 for the treatment of CLL patients.

Keywords: Chronic lymphocytic leukemia, PF-06747143, CXCR4, CXCL12, Chemokine, ADCC, CDC, Cell death, Reactive oxygen species

\footnotetext{
* Correspondence: flavia.pernasetti@pfizer.com; jecastro@ucsd.edu

${ }^{\dagger}$ Equal contributors

${ }^{3}$ Oncology Research \& Development, Pfizer Worldwide Research \&

Development, 10646 Science Center Drive, San Diego, CA 92121, USA

${ }^{1}$ Moores Cancer Center, University of California San Diego, 3855 Health

Science Drive, La Jolla, CA 92093-0820, USA

Full list of author information is available at the end of the article
} 


\section{Background}

CXCR4 (chemokine C-X-C motif receptor 4), also known as CD184, is a chemokine $\mathrm{G}$ protein coupled receptor [1], expressed in different cell types, including normal B cells [2-6]. CXCR4 is overexpressed in a variety of cancers including chronic lymphocytic leukemia (CLL), acute myeloid leukemia (AML), myeloma, lymphomas, and solid tumors [7]. CXCL12 (chemokine C-X-C motif ligand 12), also known as stromal cell-derived factor 1 (SDF-1), is CXCR4 sole ligand. It is a homeostatic chemokine [8], highly expressed in the lymph nodes, bone marrow (BM), liver, and lung [9]. CXCL12 regulates hematopoietic cell trafficking and their homing to the BM [5]. Chemotaxis driven by CXCR4 and CXCL12 interactions has been shown to control various biological functions including cell adhesion, migration, and invasion [10].

CLL is the most prevalent adult leukemia and is characterized by accumulation of dysfunctional B-lymphocytes in the lymph nodes and BM [11]. Stromal cells secrete CXCL12 and promote B-cell progenitors and CLL cell survival through CXCR4 signaling $[8,12,13]$. Thus, activation of the CXCL12/CXCR4 axis plays an important role in stromal cell-dependent resistance to therapy in CLL patients, including cytotoxic drugs [4] or steroids [14], thereby promoting minimal residual disease [15]. These observations support the rationale for targeting CXCR4 for the treatment of CLL.

In the last decade, a number of agents targeting CXCR4 have been developed. These include small molecules, peptides, and monoclonal antibodies. The role of CXCR4 in hematopoietic stem cell (HSC) retention and trafficking led to the development of agents used for HSC mobilization. AMD3100 (Plerixafor), a small molecule inhibitor of CXCR4, was approved for mobilization of HSCs prior to autologous transplantation; however, this compound has limited application for sustained treatment due to toxicity $[16,17]$. BL-8040 (BKT140), a peptide inhibitor of CXCR4, has robust cell mobilization capacity $[18,19]$, similarly to other CXCR4-specific antagonist peptides (T-140, TN14003, TC-14012), which were shown to inhibit CXCR4CXCL12 signaling in CLL-B cells [4]. However, these peptides show limited in vivo exposures. Recently, two CXCR4 human IgG4 antagonist antibodies, ulocuplumab [20-22] and LY2624587 [23], were described. Ulocuplumab is currently in phase 1 clinical studies, and it was shown to have prolonged pharmacokinetic exposure compared to small molecules or peptide inhibitors [20-22].

PF-06747143 is a novel and potentially first in class humanized IgG1 anti-CXCR4 antibody that recently entered into clinical studies (NCT02954653). Here, we show that it potently binds to CXCR4 and inhibits CXCL12-driven calcium flux. Moreover, it induces cell death in malignant CLL-B cells via two main mechanisms of action: (1) bivalency-dependent mechanism, involving generation of reactive oxygen species (ROS) and independent of caspases and (2) Fc region-driven cytotoxicity, including complement-dependent cytotoxicity (CDC) and antibodydependent cell-mediated cytotoxicity (ADCC) activity. Importantly, we show that PF-06747143 triggers cell death in B-CLL patient-derived primary leukemia cells, in spite of the presence of stromal cells, mimicking the leukemia microenvironment in vitro. The antibody also synergizes with conventional CLL treatment agents such as bendamustine, rituximab, fludarabine (F-ara-A), and ibrutinib, significantly improving their cytotoxicity in combination. Furthermore, we show that PF-06747143 inhibits tumor burden and improves survival as a monotherapy or in combination with bendamustine, in a CLL xenograft tumor model. Based on these unique mechanisms of action, PF-06747143 has a promising therapeutic potential in CLL patients and other hematological malignancies dependent on the CXCR4 axis.

\section{Methods}

\section{Isolation of PBMCs from CLL patients}

The CLL-B cells were collected from blood samples at the Moores-UCSD Cancer Center in compliance with the Declaration of Helsinki and after approval of the UC San Diego Institutional Review Board (IRB) [24].Peripheral blood mononuclear cells (PBMC) from CLL patients were isolated using Ficoll-Hypaque gradient density centrifugation (Cat\# 17-1440-03, GE Healthcare Life Science). For caspase activation assays, the CLL-B cells were purified by positive selection using Dynabeads CD19 pan B (Cat\# 11143D, Invitrogen) and DETACHaBEAD CD19 (Cat\# 12506D, Invitrogen) according to the manufacturer's protocol. For the other assays, fresh or frozen PBMCs were used and cells were stained with CD19/CD5 antibodies for detection of double positive CLL-B cells.

\section{CLL-B cells co-culture to mimic CLL microenvironment}

Primary leukemic cells from CLL patients were cultured in RPMI supplemented with $10 \%$ heat-inactivated FBS (fetal bovine serum, Catalog \# FB-02, Omega Scientific, Tarzana, CA) and $1 \%$ antibiotic at a density of $3 \times 10^{5}$ cells per milliliter at $37{ }^{\circ} \mathrm{C}$ and $5 \% \mathrm{CO}_{2}$. The cells were either cultured in 96-well round bottom plates (Catalog \# 3596, Corning, NY) alone or co-cultured with NK-tert stromal cells (RIKEN, Yokohama, Japan) at a ratio of 20:1 (CLL: stroma-NK-tert) in RPMI with 1\% PennStrep and 10\% FBS [25].

\section{CXCR4 expression by flow cytometry}

The CXCR4 phenotyping of CLL-B, stroma-NK-tert, normal $\mathrm{B}$, and $\mathrm{T}$ cells was done by flow cytometry using a 1:50 dilution with rat anti-human CD184 (CXCR4) PE Mab (Catalog \# 551966, clone:2B11, BD Biosciences). 
The isotype control antibody was $\mathrm{PE}$ Rat $\mathrm{IgG}_{2 \mathrm{~b}}$, $\mathrm{K}$ (Catalog \# 12-4031-83, clone: eB149/10H5, eBioscience).

\section{CXCR4 antibody generation}

The parental CXCR4 antagonist antibody, m15, was derived from immunization of Balb/c mice with $\mathrm{CHO}$ cells transfected with human CXCR4. The heavy and light chain variable domains of $\mathrm{m} 15$ were then cloned into human IgG1 or hinge stabilized IgG4 and light $\mathrm{k}$ backbone, to generate chimeric m15-IgG1 and m15-IgG4. m15 was subsequently humanized by CDR grafting/affinity maturation and cloned into human $\mathrm{IgG} 1 / \mathrm{K}$ constant domains to create PF-06747143.

\section{Binding kinetics and affinity}

Experiments were performed on a Biacore ${ }^{\mathrm{TM}} \mathrm{T} 200$ surface plasmon resonance biosensor (GE Life Sciences). The binding to human CXCR4 was determined using human CXCR4-enriched lipoparticles (Integral Molecular) compared to null particles. Lipoparticles were diluted into $10 \mathrm{mM}$ HEPES, $150 \mathrm{mM} \mathrm{NaCl}, 1 \mathrm{mg} / \mathrm{mL}$ BSA, pH 7.4 buffer to concentrations between 0.015 to 0.04 units $/ \mathrm{mL}$ and captured for 5 min onto flow cells. A threefold dilution series of Fab was evaluated and dissociation was monitored for $10 \mathrm{~min}$. The data were fit to a 1:1 Langmuir with mass transport model using Biacore T200 Evaluation Software Version 2.0.

\section{PF-06747143 binding to tumor cells by flow cytometry}

Cell suspensions ( $n=3$ /group) were stained with $20 \mu \mathrm{g} /$ $\mathrm{mL}$ of either a human IgG1 к Phycoerthrin (PE)-labeled antibody (isotype control) (Southern Biotech) or with PF06747143 PE-conjugated antibody, labeled using the SiteClick $^{\text {Tw }}$ R-PE Kit (Molecular Probes, Life Technologies). Flow cytometric acquisition and analysis was conducted using FACS LSRII ${ }^{\text {rm }}$ flow cytometer (Beckman Dickinson).

\section{Calcium flux functional assay}

The ability of PF-06747143 or m15-IgG1 to inhibit CXCL12-induced calcium flux was evaluated in human $\mathrm{T}$ cell leukemia Jurkat cells using the Fluo-NW Calcium assay kit (Life Technologies). Cells were plated in 384-well plates at 70,000 cells per well in quadruplicates and incubated with m15-IgG1 parent antibody and PF-06747143, upon stimulation with CXCL12 at $8 \mathrm{nM}$ (EC80) (Invitrogen), for $110 \mathrm{~min}$. Calcium flux was then measured for $95 \mathrm{~s}$ using a FLIPR Tetra (Molecular Devices).

\section{Cell death}

Cell death was evaluated by flow cytometry analysis using CD19/CD5/Annexin V antibodies [26]. Specific induced cell death (SICD) calculation was used in order to discriminate the antibody/compound-specific induced cell death from background or spontaneous cell death observed in the vehicle-treated groups. The calculation of $\%$ SICD was performed using the following formula: $\% \mathrm{SICD}=$ (Compound-induced cell death - Vehicle spontaneous cell death $) /(100$ - Vehicle spontaneous cell death) $\times 100$.

\section{Cell death in combination with CLL standard of care agents}

m15-IgG1 was tested in combination with different standard of care (SOC) agents currently used for treatment of CLL. F-ara-A, bendamustine, rituximab, and ibrutinib were evaluated in combination with $200 \mathrm{nM}$ of m15-IgG1. CLL-B cells were treated for $48 \mathrm{~h}$ at $37^{\circ} \mathrm{C}$ either cultured alone or co-cultured with stroma-NK-tert cells. The combination data and level of synergism was analyzed using CompuSyn software (ComboSyn, Inc., NJ, USA). The data derived from this analysis were expressed as combination index $(\mathrm{CI})$, which offers definition for additive $(\mathrm{CI}=1)$, synergism $(\mathrm{CI}<1)$, and antagonism $(\mathrm{CI}>1)$ in drug combination [27].

\section{Antibody-dependent cellular cytotoxicity (ADCC) assay}

For analysis of ADCC in B-CLL patient primary cells, the ADCC Reporter Bioassay kit from Promega (Catalog \#G7010) was used, per instructions from the manufacturer. The ADCC Reporter Bioassay uses engineered Jurkat cells stably expressing the FcyRIIIa receptor, V158 (high affinity) variant, and a NFAT (nuclear factor of activated T cells) pathway response element driving expression of firefly luciferase as effector cells. The transfected Jurkat cell line was grown in RPMI containing G-418 sulfate solution (Catalog \# V8091) and hygromycin (Catalog \# 10687010, $50 \mathrm{mg} / \mathrm{mL}$ solution). The ADCC buffer (99.5\% RPMI 1640 with Lglutamine and $0.5 \%$ super low IgG FBS) was prepared using RPMI supplemented with super low IgG defined fetal bovine serum (catalog \# SH30898, Hyclone). The luciferase assay system was used as a readout (Catalog \# G7940, Promega). Different concentrations of antibodies IgG1 control, PF-06747143, rituximab and obinutuzumab were added to the effector/target cell 1:1 ratio mixtures. A total of 75,000 for effector and target cells were incubated for $6 \mathrm{~h}$ at $37^{\circ} \mathrm{C}$ in a humidified $\mathrm{CO}_{2}$ incubator. Following incubation, the plate was equilibrated to ambient temperature for $15 \mathrm{~min}$. Bio-Glo ${ }^{\text {Txt }}$ luciferase assay reagent was added and incubated at room temperature for $30 \mathrm{~min}$. The luminescence was detected using an Infinite 200 Microplate Reader (Tekan), and the results are expressed in relative light units (RLU).

ADCC activity of PF-06747143 and m15-IgG1 parent antibody was evaluated in JVM-13 CLL tumor cell line, in presence of the NK-92 FcyRIIIA 158V (NK92 158V) cell line as effector cells (Conkwest). Antibodies were incubated for $4 \mathrm{~h}$, with tumor cells and effector cells (1:10 ratio) ( $n=4 /$ group). ToxiLight bioluminescent cytotoxicity assay (Cat \# LT07-117 Lonza) was used to detect cell lysis. 


\section{CDC assay}

PF-06747143 was added to CLL-B cells $\left(1 \times 10^{6} / \mathrm{mL}\right)$ in RPMI media with $5 \%$ active human serum $[28,29]$ or inactivated human serum, which was incubated at $56{ }^{\circ} \mathrm{C}$ for $30 \mathrm{~min}$. The heat-inactivated/normal human serumtreated cells were incubated for $4 \mathrm{~h}$ at $37{ }^{\circ} \mathrm{C}$ with increasing concentrations of PF-06747143. Cytotoxicity was determined by flow cytometry using CD19/CD5/ Annexin V staining. \% SICD was calculated according to the following formula: $100 \times(\%$ viable cells with inactivated serum - \% viable cells with native serum $) /(\%$ viable cells with inactivated serum).

\section{Inhibition of actin polymerization}

Cytoskeletal reorganization (F-actin polymerization) was evaluated in CLL samples activated by CXCL12 and treated with PF-06747143 or control agents [4].

\section{Inhibition of migration of cells in a transwell assay} PF-06747143 was assessed for its ability to inhibit CXCL12-induced chemotaxis in primary CLL-B cells derived from CLL patients using a transwell migration assay [30].

\section{Caspase activity assay}

To evaluate the mechanism of cell death induced by PF06747143, CLL-B cells were purified from patient-derived PBMCs and tested for caspase activation including caspases 3, 8, and 9 using the ApoTarget Caspase Colorimetric Protease Assay Sampler kit (Cat \# KHZ1001, Invitrogen, Frederick, MD) according to the manufacturer's instructions. Z-VAD-FMK, a caspase inhibitor (Cat \# G7231, Promega Corporation), was used as control [31].

\section{Detection of reactive oxygen species (ROS) by flow cytometry}

CLL-B cells were seeded at $2.5 \times 10^{5} / \mathrm{mL}$ in RPMI media and treated with antibodies for $4 \mathrm{~h}$ at $37{ }^{\circ} \mathrm{C}$ and $5 \% \mathrm{CO}_{2}$ in 24-well plates. The generation of ROS was detected using dihydroethidium (HE) staining (Catalog \# D1168, SigmaAldrich, St. Louis, MO) as described previously [32]. The samples were then analyzed by flow cytometry followed by data analysis using FlowJo software.

\section{In vivo efficacy study}

JVM-13 tumor cell line [33, 34], purchased from ATCC, was stably transfected with the luciferase gene. The cells were cultured in RPMI media with $10 \%$ FBS. To establish a JVM-13 disseminated model, $1 \times 10^{6}$ cells per mouse were implanted via tail vein injection in female SCID beige mice (Charles River). Tumor burden was monitored via bioluminescence imaging (BLI) (IVISÒ 200) throughout the study. When the tumor burden (mean BLI) reached $7.2 \times 10^{6}$ photons/s, on day 19 , mice were randomly assigned into four groups and treated with (1) IgG1 negative control Ab or (2) PF-06747143, dosed subcutaneously at $10 \mathrm{mg} / \mathrm{kg}$, once a week, for a total of 6 doses; (3) bendamustine, dosed intraperitoneally at $30 \mathrm{mg} / \mathrm{kg}$, on days 19 and 20, followed by another 2-day treatment cycle 28 days later; and (4) combination of PF-06747143 and bendamustine. Mice were euthanized according to the IACUC guidelines once they developed disease-related symptoms such as hind leg paralysis.

\section{Statistical analysis}

The statistical analysis was carried out using GraphPad Prism software (v. 5.0c; San Diego, CA). The statistical differences for the mean values were analyzed using one way ANOVA and are indicated with *, $p<0.05$;*, $p<0.01$; **, $p<0.001$; and ****, $p<$ 0.0001 . Tumor model survival analysis was performed using Kaplan-Meier followed by a long-rank (Mantel-Cox) test.

\section{Results}

Expression of CXCR4 in CLL, normal B cells, stroma-NKtert, leukemia, and lymphoma cell lines

Expression of CXCR4 was evaluated by flow cytometry in primary B-CLL cells from patients, as well as normal $\mathrm{B}, \mathrm{T}$, and stroma-NK-tert cells. A representative example of CXCR4 staining is shown in Fig. 1a. CXCR4 expression was higher in CLL-B cells compared to normal $\mathrm{B}$ and T cells. We then assessed CXCR4 expression levels in high- and low-risk CLL patient groups. CLL patients were stratified into high risk (ZAP-70 $\geq 20 \%$, $\operatorname{IgVH} \geq 98 \%$ homology) and low risk (ZAP-70 $<20 \%$, IgVH $<98 \%$ homology) based on ZAP-70 expression and IgVH homology status [35]. The average mean fluorescence $(\triangle \mathrm{MFI})$ for CXCR4 expression in CLL samples ranged from 263.73 to 2401.7 , regardless of high- or low-risk status. The $\triangle M F I$ in high-risk patients was $1497.23 \pm 195.89$ and in low-risk patients, it was $1533.73 \pm 178.54$. Upon comparison between low- and high-risk CLL patients, no significant difference in CXCR4 expression levels was observed $(p=0.8601)$. Overall, CXCR4 expression was significantly higher in CLL-B cells as compared to normal B or T cells (10and 22-fold, respectively; $p<0.0001$ ) (Fig. 1b). Furthermore, CXCR4 expression in leukemia and lymphoma cell lines including K562, MEC1, Namalwa, Raji, JeKo1 , and Jurkat showed a broad range of expression levels, with $\triangle$ MFIs of $-2.35,0.01,73.41,526.72,725.45$, and 1358.5, respectively (Additional file 1: Figure S1).

\section{PF-06747143 and the parental $\mathrm{m} 15$ antibody bind CXCR4 with high selectivity and affinity}

To determine the binding specificity and affinity of PF06747143 and its parental antibody m15 to human (h) 

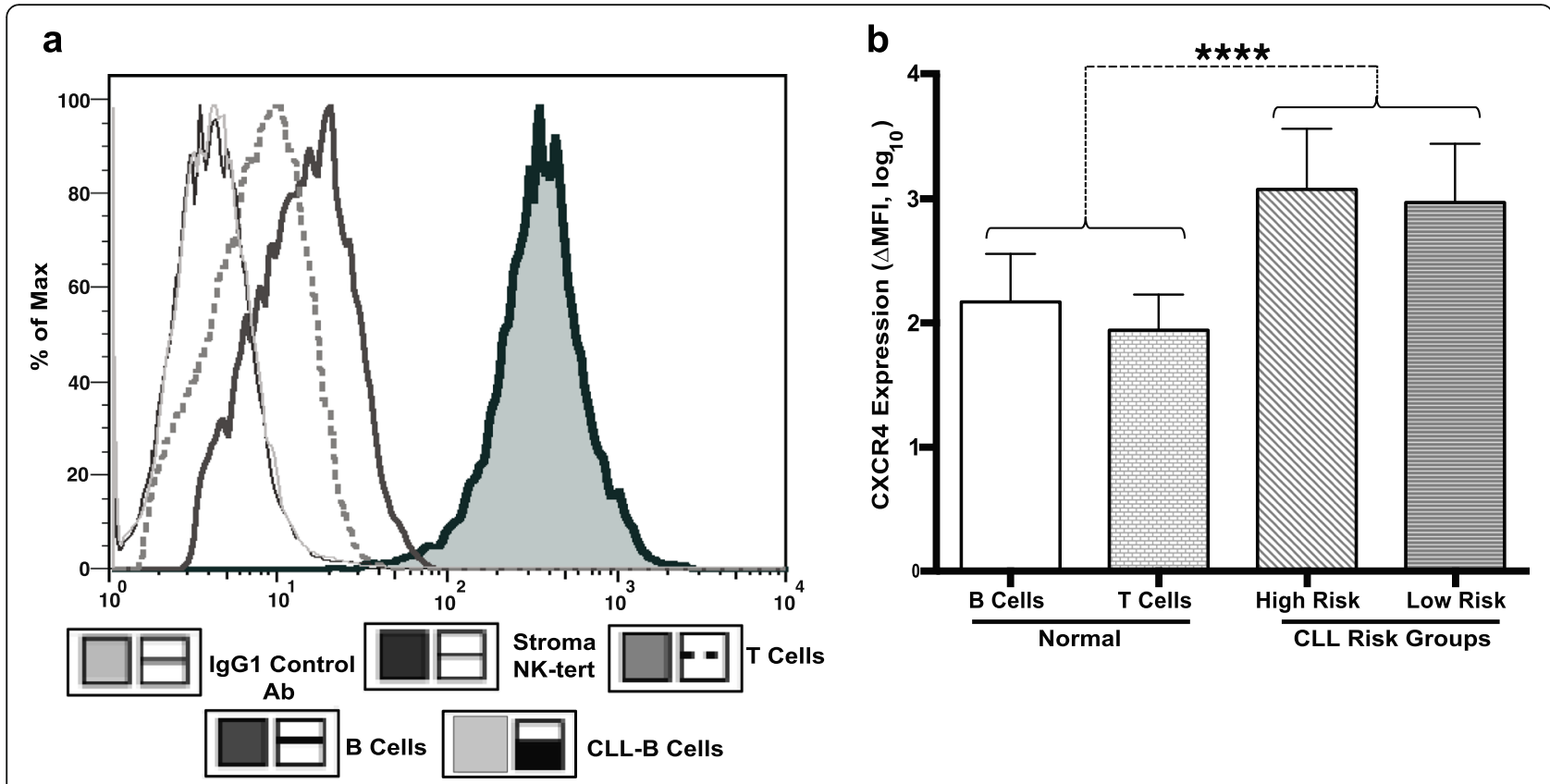

Fig. 1 Expression of CXCR4 receptor in CLL-B, normal B, T, and stroma-NK-tert cells. a CXCR4 expression was assessed by surface staining using an anti-CXCR4 antibody in CLL-B, normal B, T, and stroma-NK-tert cells. A representative panel is shown. $\mathbf{b}$ CXCR4 expression was evaluated by flow cytometry in CLL patient cells with high-risk and low-risk characteristics ( $n=20$ per group) and in normal B and T cells obtained from healthy donors ( $n=5$ per group). The figure shows the mean fluorescence intensities (MFI) \pm standard deviation (SD) of the samples analyzed in duplicate for CXCR4 expression in each cell type. Statistical significance was determined by using Bonferroni's correction test for multiple comparison tests, where $*^{* * * * *}$, and ${ }^{* * * *}$ represent $p<0.05 ; p<0.01, p<0.001$, and $p<0.0001$, respectively

CXCR4, we employed surface plasmon resonance. The monovalent Fab fragments of each antibody were incubated with hCXCR4-enriched lipoparticles. The apparent equilibrium dissociation constant $\left(K_{D}\right)$ was $0.36 \mathrm{nM}$ for PF-06747143 and $0.67 \mathrm{nM}$ for the parental antibody, m15, indicating that both antibodies have potent and comparable affinity to hCXCR4 (Table 1). None of the Fabs bound to lipoparticles lacking hCXCR4 (data not shown). In a separate experiment, PF-06747143 was fluorescently labeled (PF-06747143-PE) and binding to $\mathrm{CHO}$ cells expressing hCXCR4 (CHO-hCXCR4) was compared to binding to cells transfected with empty vector (CHO-parental). PF-06747143-PE bound specifically to $\mathrm{CHO}$ cells expressing hCXCR4 (CHO-hCXCR4) but not to the $\mathrm{CHO}$-parental cells (Fig. 2a), demonstrating selective binding to CXCR4-expressing cells.

Table 1 Biosensor kinetics and affinity measurements

\begin{tabular}{llllc}
\hline Clone name & $k_{a}(1 / \mathrm{Ms})$ & $k_{d}(1 / \mathrm{s})$ & $t_{1 / 2}(\mathrm{~min})$ & $k_{D}(\mathrm{nM})$ \\
\hline m15 Fab & $2.1 \times 10^{6}$ & $1.4 \times 10^{-3}$ & 8.3 & 0.67 \\
PF-06747143 Fab & $8.0 \times 10^{5}$ & $2.9 \times 10^{-4}$ & 40 & 0.36
\end{tabular}

Binding affinities of the Fab regions derived from PF-06747143 and its parent antibody $\mathrm{m} 15$ were measured by surface plasmon resonance

$k_{a}$ kinetic association constant, $k_{d}$ kinetic dissociation constant, $t_{1 / 2}$ dissociation half-life, $K_{D}$ equilibrium dissociation constant
PF-06747143 and the parental antibody m15-IgG1 inhibit CXCL12-induced calcium flux

Calcium flux is triggered upon activation of CXCR4 by its ligand, CXCL12. We next evaluated the ability of PF06747143 and its parental antibody, m15, expressed as a chimeric human IgG1 antibody (m15-IgG1), to inhibit calcium flux induced by CXCL12. The Jurkat T cell leukemia line, which expresses high levels of CXCR4 (Additional file 1: Figure S1), was incubated with CXCL12 $\left(\mathrm{EC}_{80}\right.$ at $8 \mathrm{nM}$ ) to stimulate calcium flux. A titration of PF06747143 and m15-IgG1 was performed. Both PF06747143 and m15-IgG1 blocked CXCL12-induced calcium flux in a dose-dependent manner, with similar $\mathrm{IC}_{50 \mathrm{~s}}$ of 1.41 and $1.13 \mathrm{nM}$, for PF-06747143 and m15-IgG1, respectively. These results show that both CXCR4 antibodies have potent and comparable CXCL12 antagonistic activity (Fig. 2b).

Next, we evaluated if bivalency was required for PF06747143 to inhibit calcium flux. To this end, a bivalent form of PF-06747143, which has no constant Fc region $\left[\mathrm{F}\left(\mathrm{ab}^{\prime}\right)_{2}\right]$, and a monovalent form of the antibody [Fab], were generated and compared to PF-06747143, which is a bivalent full-length antibody (PF-06747143 FL). (Additional file 2: Figure S2). Similar CXCL12-induced calcium flux inhibition was observed for all three forms of PF-06747143 tested, indicating that the functional CXCL12 antagonistic 


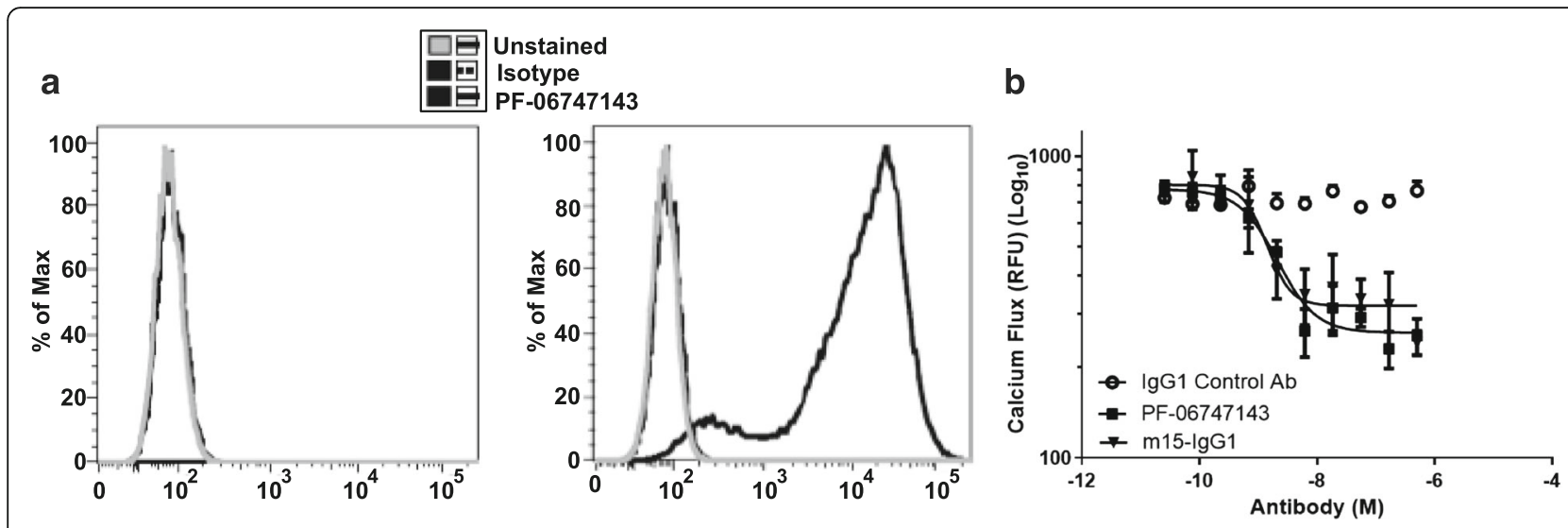

Fig. 2 PF-06747143 binds specifically to human CXCR4-expressing cells and blocks CXCL12-induced calcium flux. a CHO-parental and CHO-hCXCR4 cell lines were exposed to $20 \mathrm{\mu g} / \mathrm{mL}$ of either a human lgG1 k-PE antibody (isotype control) or PF-06747143-PE and analyzed by flow cytometry. b Calcium flux assay was performed in human T cell leukemia Jurkat cells incubated with PF-06747143, m15-lgG1, or isotype control lgG1 antibody in presence of CXCL12 at $8 \mathrm{nM}$. Experiment was performed in quadruplicates. Shown are mean intracellular calcium concentrations in relative fluorescence units (RFU). \pm standard error of the mean (SEM)

activity is not dependent on bivalent binding or Fc constant region of the antibody.

\section{The CXCR4 antibody induces cell death in CXCR4- expressing CLL patient cells}

m15-IgG1 was evaluated for its ability to trigger cell death upon binding to primary CLL-B cells expressing CXCR4 or to the MEC1 (CLL) cell line, which has no detectable CXCR4 expression $(\triangle M F I=0.01)$ (Fig. 3a). Cells were incubated with increasing concentrations of m15-IgG1 or control IgG1 antibody and analyzed for cell death using flow cytometry. CLL-B cells underwent cell death upon treatment with m15-IgG1 $(2-2000 \mathrm{nM})$ in a dose-dependent manner, while MEC1 cells did not show evidence of cell death, even in presence of high concentrations of the antibody (Fig. 3b), indicating that the CXCR4 antibody cell death is CXCR4 expression dependent.

\section{The CXCR4 antibody induces cell death in spite of the presence of stromal cells}

To determine whether m15-IgG1 could induce cell death in presence of stromal cells, CLL-B cells were cultured with stroma-NK-tert cells in presence of increasing concentrations of m15-IgG1 and cell death was evaluated after $48 \mathrm{~h}$. F-ara-A, an agent that inhibits DNA synthesis and is a cornerstone for the treatment for CLL patients [36] as well as AMD3100, a CXCR4 small molecule inhibitor [37], were evaluated for comparison. m15-IgG1 induced cell death of leukemia cells cultured either alone or in presence of stromal cell support (stroma NK-tert cells), demonstrating the ability of the antibody to induce cell death in presence of stromal cells (Fig. 3c). Similar results were observed for various B-CLL patients (Additional file 3: Figure S3).
Moreover, m15-IgG1 was more potent at inducing cell death than F-ara-A $(p<0.0001)$ in CLL-B cells (Fig. 3c). AMD3100, which binds and inhibits signaling through CXCR4, did not induce cell death in CLL-B cells (Fig. 3c), indicating that binding and inhibition of the CXCR4 pathway is not sufficient to trigger cell death.

Next, we sought to determine whether the Fc constant region or backbone of the CXCR4 antibody played a role in the ability to induce B-CLL cell death. We compared m15IgG1 to m15-IgG4, which is an antibody with the same antigen-binding regions from $\mathrm{m} 15$-IgG1, cloned in a human IgG4 constant region. Results from these studies (Additional file 4: Figure S4) demonstrate that both antibodies are capable to induce cell death in CLL-B cells to similar degrees, with no significant difference between $\mathrm{m} 15$-IgG1 and m15IgG4, cultured alone or co-cultured with stroma cell support $(p>0.05)$. This indicates that the antibody constant region does not play a role in this cell death mechanism.

\section{The CXCR4 antibody induces B-CLL cell death independently} of CLL risk factor and spares normal B and T lymphocytes

In addition to the high-risk and low-risk prognosis markers ZAP70 and IgVH, the 17p deletion in the TP53 gene is also considered a strong independent adverse prognostic factor for survival and is associated with the short median treatment-free survival, in CLL patients with CLL [38].

To evaluate the ability of the CXCR4 antibody to induce cell death in leukemia cells from CLL patients with high risk (CLL-HR), low risk (CLL-LR), as well as with TP53 17pDel status, we treated samples from patients with these various genetic backgrounds with m15-IgG1 and evaluated cell death after $48 \mathrm{~h}$ of culture. Similar levels of cell death were induced by m15-IgG1 in CLLLR, CLL-HR, TP53wt, or TP53mut/Del(17p) patient samples (Fig. 3d). This indicates that low-risk, high-risk, or 


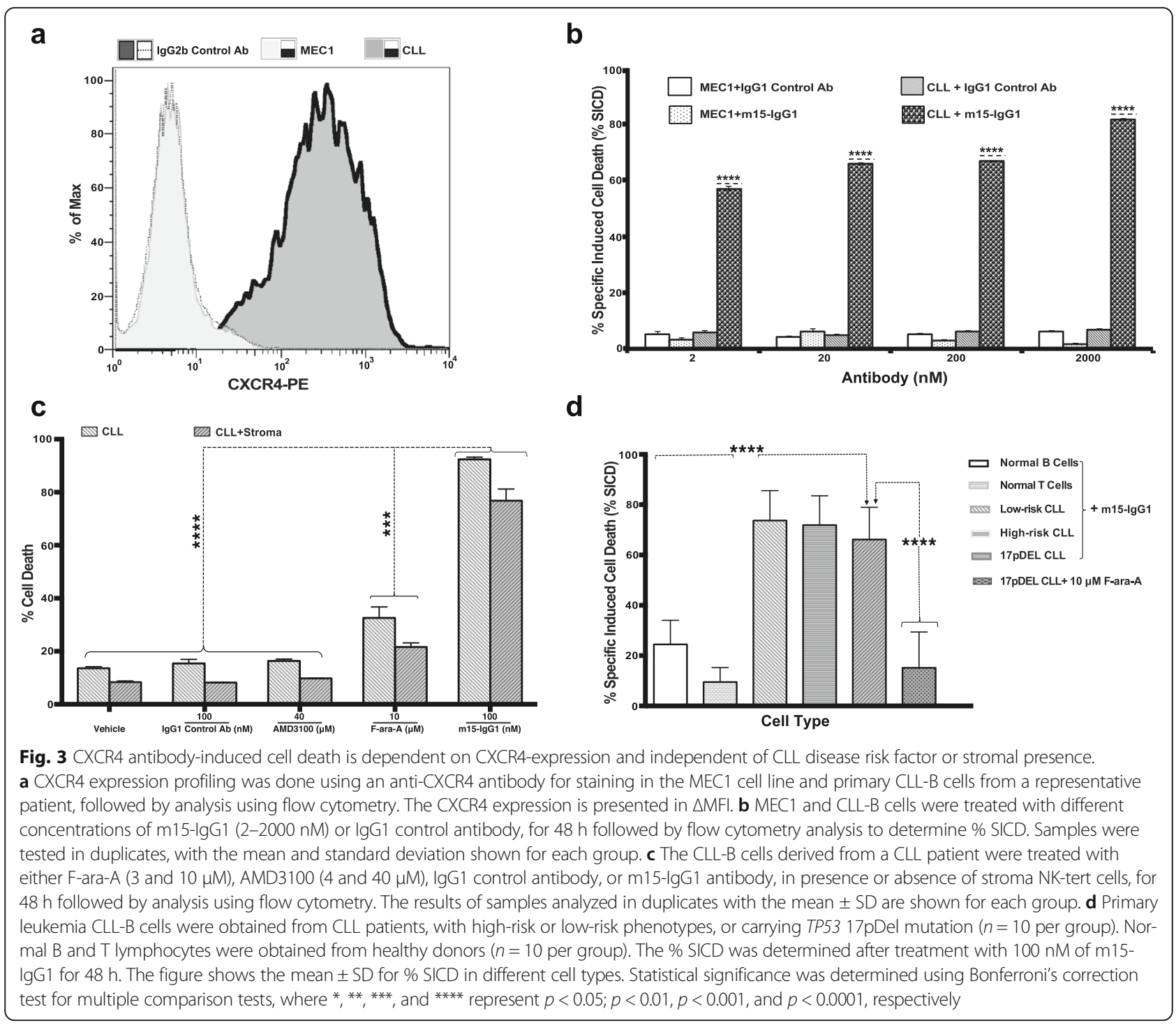

TP53 statuses are not factors for sensitivity to m15-IgG1. Moreover, m15-IgG1-induced cell death was significantly higher than that with F-ara-A $(p<0.0001)$, which is known to be a TP53-dependent chemotherapy agent [39, 40]. F-ara-A did not induce cell death in TP53mut/Del(17p) even at supra-physiological concentrations $(>3 \mu \mathrm{M})$.

Importantly, m15-IgG1 induced significantly lower levels of cell death in normal B and T lymphocytes compared with CLL samples $(p<0.0001)$ (Fig. 3d). These data suggest that m15-IgG1-induced cell death is dependent on the level of CXCR4 expressed in the cells.

To characterize the kinetics of cell death induced by the CXCR4 antibody, a washout experiment was carried out, where CLL-B cells were incubated with m15-IgG1 (200 nM) for increasing lengths of time, after which the antibody was washed out. Readouts were performed $48 \mathrm{~h}$ post-treatment initiation. Cell death was observed as early as $3 \mathrm{~h}$ and continued to increase until $48 \mathrm{~h}$ after m15IgG1 was removed (Additional file 5: Figure S5). This effect was also independent of presence of stromal cells.

\section{The CXCR4 antibody synergizes with CLL standard of care agents}

To determine if the CXCR4 antibody could offer additional benefit to available therapies, the m15-IgG1 antibody was evaluated in combination with SOC agents currently used in the treatment of CLL patients in the clinic. The percent cell death combinatorial effect was evaluated in both high-risk (HR) and low-risk (LR) CLL patient cells, in presence or absence of stromal cell support (Fig. 4). Overall, the presence of stromal cells did not appear to be a significant factor in the ability of m15-IgG1 to synergize with CLL SOC agents. Rituximab, an antiCD20 antibody $[41,42]$, was tested at 2,10 , and $30 \mu \mathrm{g} /$ 


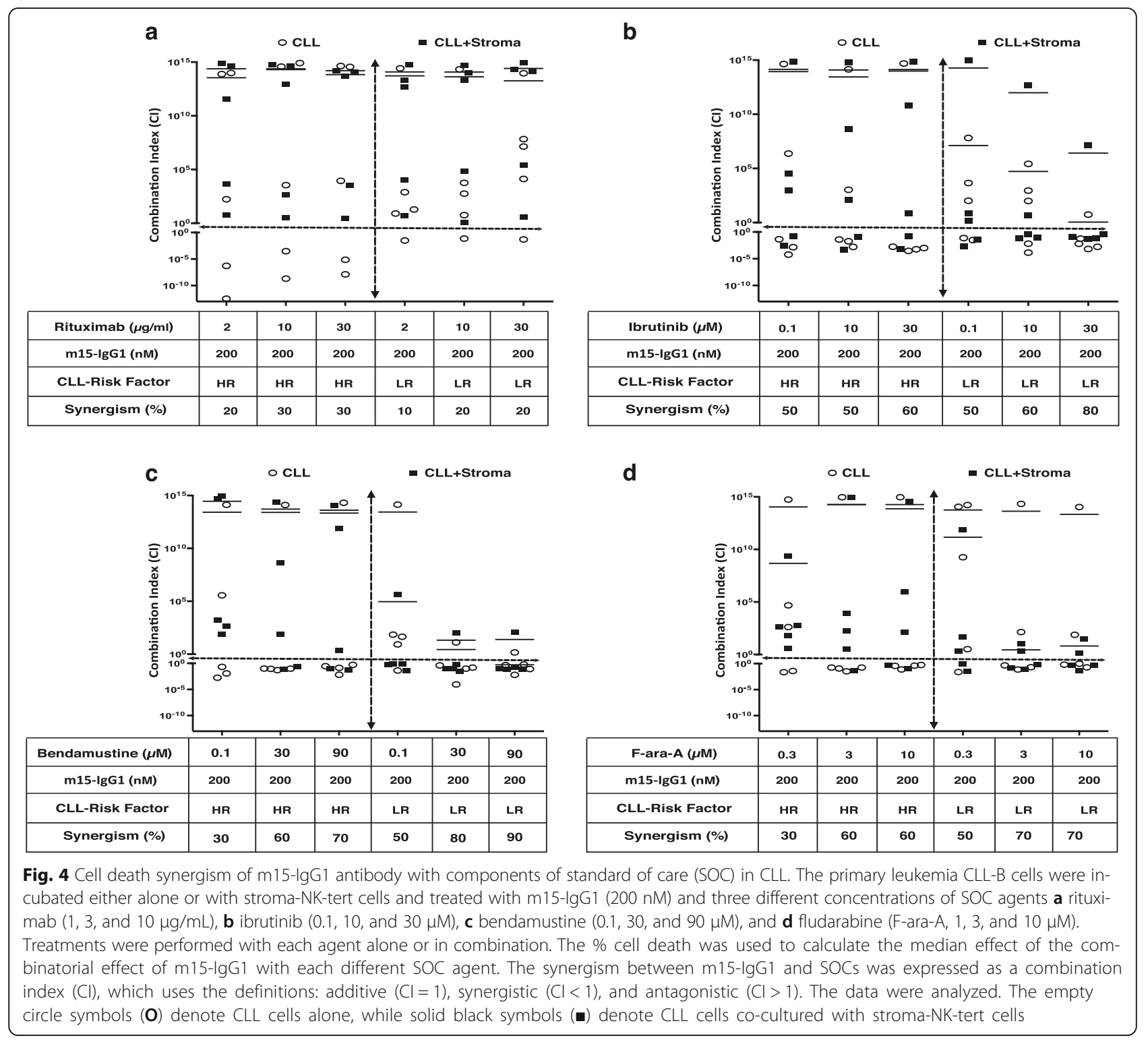

$\mathrm{mL}$. Synergistic cell death responses, in the range of 10$30 \%$, were observed for both low- and high-risk patients, for all rituximab doses tested (Fig. 4a). Ibrutinib (IBM) or imbruvica, a BTK inhibitor [43], was tested at $0.1,10$, and $30 \mu \mathrm{M}$, and synergism was observed in $50-60 \%$ of high-risk patients and $50-80 \%$ of low-risk patients samples (Fig. 4b). Bendamustine, a chemotherapy agent derived from nitrogen mustard, commonly used in the treatment of CLL and lymphomas [44], was tested at $0.1,30$, and $90 \mu \mathrm{M}$. Synergistic effects were noted in $30-70 \%$ of high-risk samples and in $50-90 \%$ of low-risk patients (Fig. 4c). F-ara-A was tested at 0.3, 3, and $10 \mu \mathrm{M}$ doses. Synergistic effects were observed in 30 $60 \%$ of high-risk and in $50-70 \%$ of low-risk patient samples (Fig. 4d).
m15-IgG1 and PF-06747143 induce similar CLL cell death To establish if the humanized IgG1 CXCR4 antibody PF06747143 and its parent antibody m15-IgG1 had comparable cell death activity, we performed a cell death study in leukemic B cells derived from B-CLL patient, comparing both antibodies. As shown in Fig. 5a, activity of both antibodies was very similar, with cell death induced at doses as low as $10 \mathrm{nM}$ by both antibodies. Both antibodies induced similar degree of cell death, regardless of the presence of stromal cells.

Intact antibodies possess two binding regions, which allow for binding to two epitopes at the same time. To determine if binding to two epitopes (bivalency) was required for PF-06747143 to induce cell death, the following forms of the PF-06747143 were compared: bivalent full- 


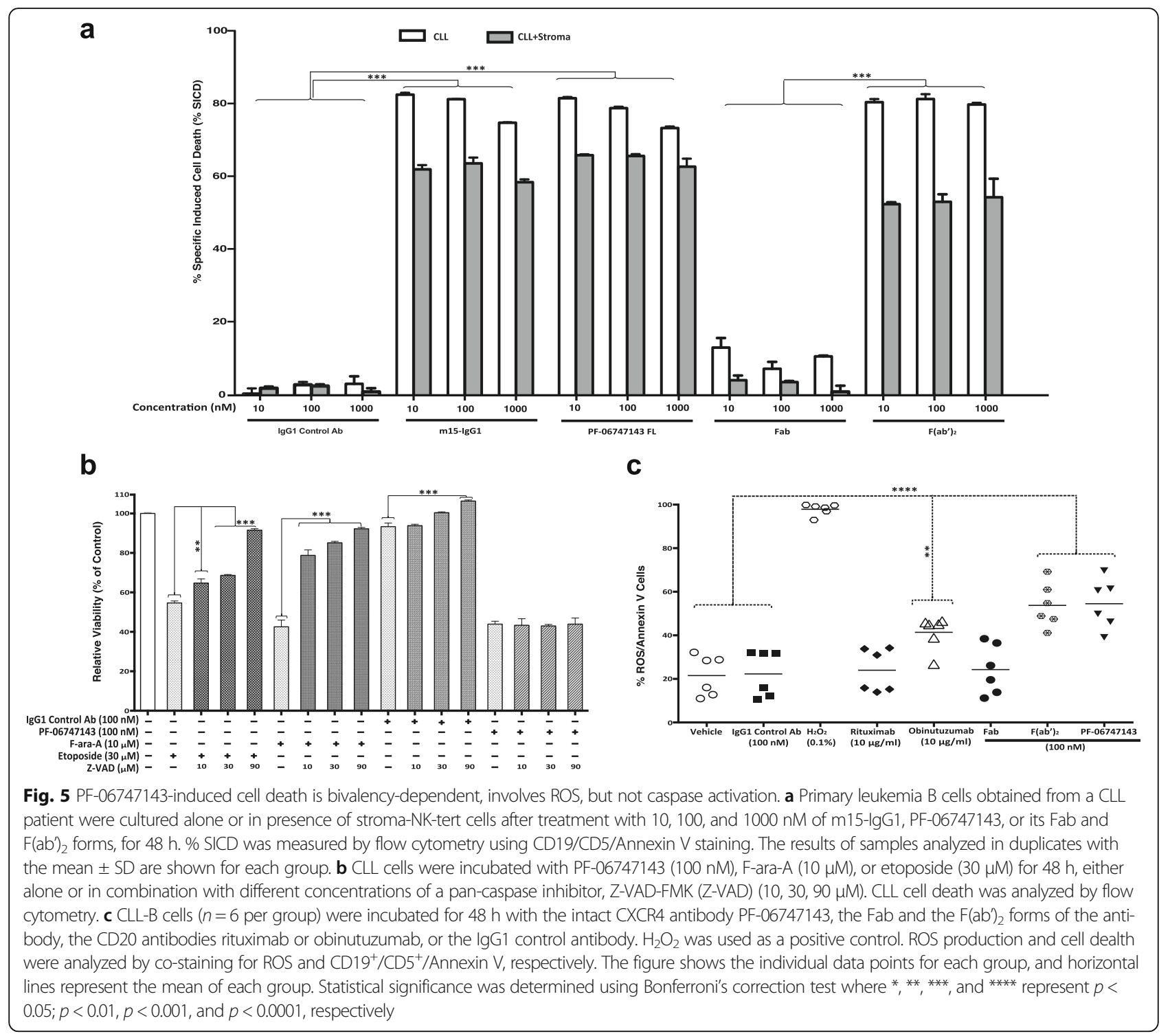

length (PF-06747143 FL), bivalent with no Fc region $\left(\mathrm{F}(\mathrm{ab})_{2}\right)$, and monovalent (Fab) (Fig. 5a). Comparable cell death (SICD) activity for the F(ab') ${ }_{2}$ form of PF-06747143 antibody and the intact antibody (FL) was observed, while the Fab form of the antibody did not induce cell death. These results indicate that bivalency, or ability to bind to two epitopes at once, is required for PF-06747143 ability to induce cell death.

\section{PF-06747143 induction of cell death is caspase independent}

To determine the mechanism through which PF06747143 induced cell death, caspase activation was evaluated. PF-06747143 treatment of CLL-B cells did not induce significant activation of caspase 3,8 , and 9 in CLL-B cells (Additional file 6: Figure S6). In a follow-up experiment, Z-VAD-FMK (Z-VAD), an irreversible pan- caspase inhibitor [45, 46], rescued caspase-dependent apoptosis in CLL-B cells after treatment with etoposide or F-ara-A, which are both known to induce caspasedependent cell death. However, Z-VAD failed to rescue the CLL-B cells treated with PF-06747143, indicating that the mechanism of cell death induced by PF06747143 is caspase independent (Fig. 5b).

\section{Reactive oxygen species (ROS) production is associated with PF-06747143-induced cell death}

Antibody-induced non-apoptotic cell death in human lymphoma and leukemia cells had been previously shown to be mediated through a ROS-dependent pathway, named programmed cell death (PCD) [32]. This novel cell death mechanism does not rely on caspase activation and is dependent on homotypic cell adhesion triggered upon antibody binding, followed by actin 
redistribution, lysosome membrane permeabilization, and ROS activation. Since we showed that PF-06747143 induction of cell death is caspase independent and bivalency dependent, we hypothesized that PF-06747143 cell death mechanism might involve ROS activation. To test this hypothesis, CLL-B cells were treated with PF06747143 or controls and evaluated for ROS production after $4 \mathrm{~h}$ of treatment. CLL-B cells treated with $\mathrm{H}_{2} \mathrm{O}_{2}$, the positive control, showed high levels of ROS compared to untreated control (*****,p<0.0001), as expected. Similarly, CLL cells treated with PF-06747143 showed a rapid increase in cell death and ROS production, compared to the untreated or to the IgG1 control Ab groups (******, $p<0.0001$ ). Of note, PF-06747143 treated cells had ROS levels similar to that of the positive antibody control obinutuzumab $(p>0.05)$, which has been reported to induce PCD associated with increased ROS levels [32]. Rituximab and F-ara-A, which are not known to induce PCD, did not show a significant increase in ROS (Fig. 5c).

To determine if binding to two epitopes (bivalency) was required for PF-06747143 ability to induce cell death via ROS production, the bivalent full-length (FL), the bivalent deprived of $\mathrm{Fc}$ region $\left(\mathrm{F}\left(\mathrm{ab}^{\prime}\right)_{2}\right)$, and the monovalent $(\mathrm{Fab})$ forms of the antibody were evaluated. Comparable ROS activity was observed for the $\mathrm{F}(\mathrm{ab})_{2}$ form of PF-06747143 antibody and the intact antibody (FL), while the Fab form did not induce cell death or ROS production (Fig. 5c). These results indicate that bivalency, or ability to bind to two epitopes at once, is required for PF-06747143 to induce cell death via a mechanism that involves ROS induction.

\section{PF-06747143 inhibits F-actin polymerization in CLL cells}

Actin polymerization, or cytoskeletal reorganization, is a surrogate marker of cancer cell migration and metastatic potential, induced by the interaction of CXCR4 with CXCL12 [47]. Therefore, we sought to characterize PF06747143 role in CXCL12-induced actin polymerization in CLL-B cells. Stimulation with CXCL12 induced an average increase in actin polymerization of $450 \%$, relative to baseline (100\%) (Fig. 6a). PF-06747143 significantly inhibited actin polymerization in a dose response-dependent manner. PF-06747143 at 100 and $1000 \mathrm{nM}$ inhibited CXCL12induced F-actin polymerization to levels below baseline at $85 \%$ and $75 \%$, respectively (***\%, $p<0.0001$ ). The small molecule inhibitor of CXCR4, AMD3100, was less potent than PF-06747143 in this assay, with very limited activity at $4 \mu \mathrm{M}$, which is its clinically relevant dose. At $40 \mu \mathrm{M}$, it showed moderate inhibition of $180 \%$, which was similar to the inhibition observed at the lowest dose of PF-06747143 (10 nM) (Fig. 6a).

\section{PF-06747143 inhibits migration of CLL patient-derived cells}

Since PF-06747143 was shown to be a potent inhibitor of cytoskeletal organization, we evaluated its ability to inhibit CLL-B cell migration driven by its ligand, CXCL12, using a transwell migration assay. CXCL12 induced chemotaxis of CLL-B cells, with an average increase $>500 \%$ over baseline (Fig. 6b). PF-06747143 (10 nM-1 $\mu \mathrm{M})$ significantly inhibited cell migration in a range from 40 to $80 \%$, relative to CXCL12 induction only ( $\left.{ }^{* * * *}, p<0.0001\right)$. The effect was dose dependent. CLL-B cells treated with AMD3100 $(40 \mu \mathrm{M})$ had similar migration inhibition activity as PF06747143 lowest dose $(10 \mathrm{nM})$, but no significant effect was observed with AMD3100 at $4 \mu \mathrm{M}$ (Fig. 6b).

\section{PF-06747143 induced CLL cell death via Fc region-mediated cytotoxicity (ADCC and CDC)}

Therapeutic antibodies may rely on their constant region (Fc domain) ability to induce target cell killing via immunemediated effector functions, such as ADCC and CDC to achieve efficacy. Human IgG1 and IgG3 antibodies have Fc domain sequences that can mediate potent effector functions, while human IgG4 and IgG2 antibodies display little or no effector function [48]. PF-06747143 is a humanized IgG1 antibody; therefore, ADCC and CDC studies were performed to characterize the antibody Fc-driven cytotoxic activity on CLL patient cells.

CDC cell death mechanism depends on the interaction between active serum complement proteins with the Fc region of the antibody, upon binding to the target cells. To evaluate if PF-06747143 could induce CDC in CLL-B cells, it was tested in the presence of active or inactive serum protein. As shown in Fig. 7a, PF-06747143 significantly $(p<0.0001)$ increased cytotoxicity in presence of active complement, relative to inactive complement, and this response was antibody dose dependent.

In the case of ADCC, effector cells bind to the Fc region of the antibody, when bound to target cells, and trigger cell lysis. To characterize the Fc-mediated ADCC activity of PF-06747143, CLL-B cells were incubated with the antibody in presence of effector cells. Two therapeutic antibodies, rituximab and obinutuzumab, that bind to CD20 and rely on ADCC as their main mechanism of action were used as positive controls. As expected, rituximab and obinutuzumab induced ADCC of CLL-B cells to high levels. PF06747143 had comparable ADCC activity to that of the CD20 antibodies, inducing significant ADCC, when compared to the negative IgG1 control antibody (Fig. 7b). Taken together, these data demonstrate that PF06747143, a humanized IgG1 antibody, has strong Fcdriven cytotoxic-dependent activity, leading to elimination of CLL-B cells. 

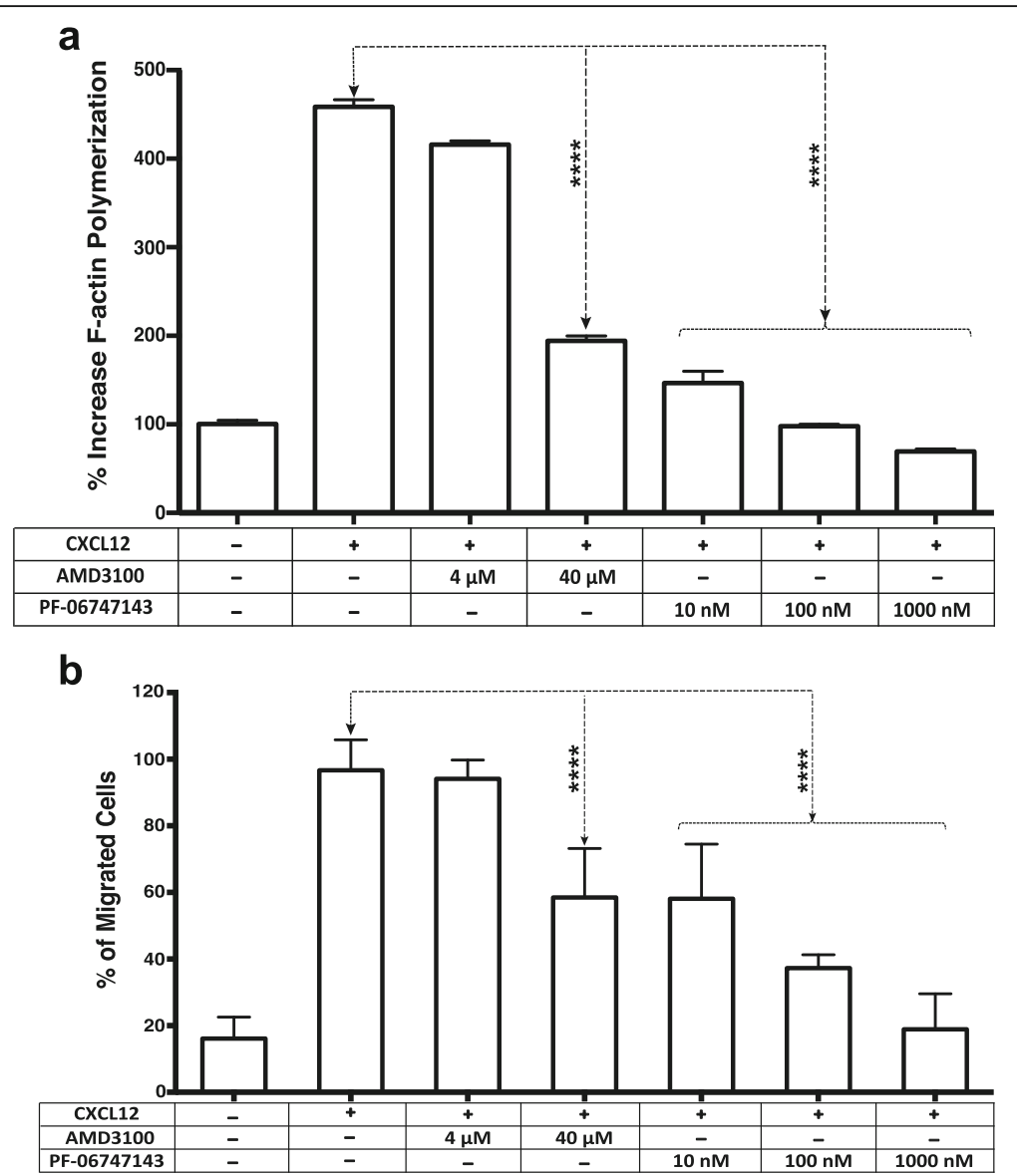

Fig. 6 PF-06747143 inhibits CXCL12-induced tumor cell actin polymerization and migration. a B-CLL patient cells were treated with no compound (negative control), AMD3100 (4 and $40 \mu \mathrm{M}$ ), or PF-06747143 (10, 100, and $1000 \mathrm{nM}$ ) prior to stimulation with CXCL12 (90 nM) for 15 s. F-actin polymerization was measured using FITC-labeled phalloidin in CD19/CD5-pre-labeled CLL patient cells. All samples are plotted relative to the mean fluorescence intensity of the negative control group, without chemokine CXCL12, set to 100\%. The results of samples analyzed in duplicates with the mean \pm SD are shown for each group. $\mathbf{b}$ CLL patient primary cells were incubated with PF$06747143(10,100,1000 \mathrm{nM})$ or $\operatorname{AMD} 3100(4$ and $40 \mu \mathrm{M})$ for $1 \mathrm{~h}$ and loaded onto a transwell chamber and incubated for $2 \mathrm{~h}$ in the presence of CXCL12 (12 nM) or media control. Cells that migrated to the lower chamber were enumerated using flow cytometry. The results of samples analyzed in duplicates with the mean \pm SD are shown for each group. Statistical significance was determined using Bonferroni's correction test

To evaluate the role of the human IgG backbone in the ADCC activity of a CXCR4 antibody, we compared PF-06747143, m15-IgG1, and m15-IgG4, generated by cloning of the m15 variable domain in a human IgG4 backbone. The ADCC assay was performed using the CLL tumor cells (JVM-13), in presence of NK92158V effector cells. The m15-IgG1 antibody showed strong cytotoxicity, when compared to no activity with the m15-IgG4 antibody (Fig. 7c). This is in agreement with the expected diminished ADCC activity of an IgG4 antibody. In addition, PF06747143 exerted similar cytotoxicity to that of $\mathrm{m} 15$ IgG1, confirming that the parent antibody (m15-IgG1) and its humanized antibody (PF-06747143) have comparable ADCC functional properties.
PF-06747143 is efficacious as a monotherapy and in combination with bendamustine in a CLL xenograft established in vivo model

To determine if PF-06747143 is effective in eliminating CLL tumor cells in vivo, a disseminated model in which the tumor cells were implanted intravenously and migrate spontaneously to various sites in the body, including the BM and lymph nodes, was used (Fig. 8). Activity of PF-06747143 was evaluated as a monotherapy as well as in combination with bendamustine, a SOC agent approved for CLL treatment. Bioluminescence imaging performed on day 26 showed that PF-06747143 treatment as a monotherapy decreased tumor burden compared to hIgG1 control Ab and bendamustine groups. A combinatorial effect was observed when PF-06747143 

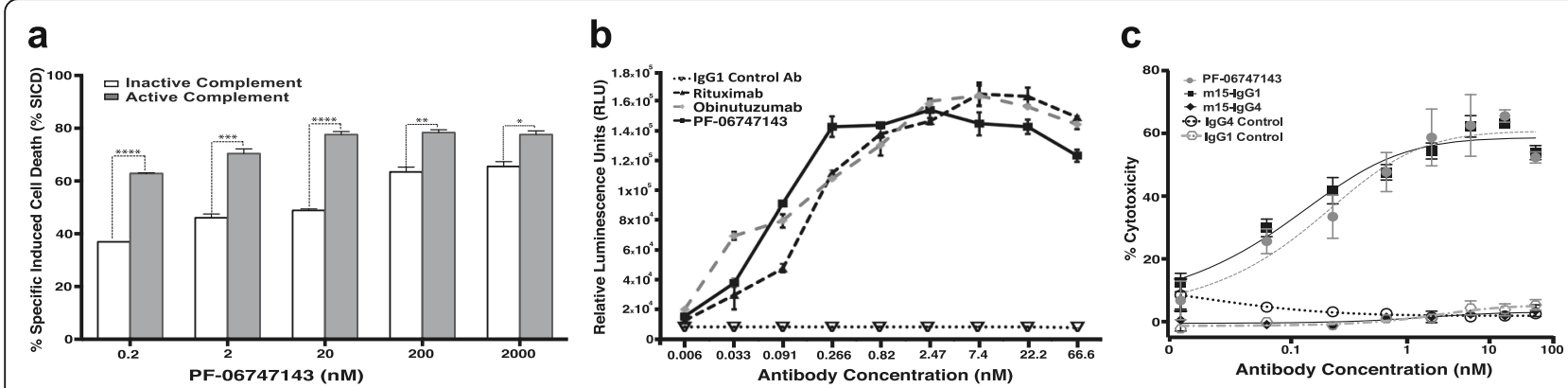

Fig. 7 PF-06747143 induces CLL-B cell killing by ADCC and CDC. a CDC assay was performed by treating CLL-B cells $\left(1 \times 10^{6} / \mathrm{mL}\right)$ with PF-06747143, in presence of complete or heat-inactivated $5 \%$ human serum. The cells were incubated for $4 \mathrm{~h}$ and the cytotoxicity was determined using flow cytometry with CD19/CD5/Annexin V staining. b The ADCC assay in patient B-CLL cells was performed using 1:1 ratio of the target/effector cell (T/E) and incubated for $6 \mathrm{~h}$ at $37^{\circ} \mathrm{C}$. The lgG1 control antibody, PF-06747143, and rituximab were tested in a 1:3 titration curve, ADCC activity was determined using Bio-Glo $\mathrm{o}^{\mathrm{TM}}$ luciferase assay, and the luminescence results are expressed in relative light units (RLU). The samples were analyzed in duplicates with the mean and SD shown for each group. The data was analyzed using Prism 4 GraphPad software. c ADCC activity was evaluated in JVM-13 tumor cells by incubating the cells with PF-06747143, m15-lgG1, m15-lgG4, or respective negative control antibodies for $4 \mathrm{~h}$, in the presence of NK92 $158 \mathrm{~V}$ effector killer cells (effector/target cell ratio 10:1). Cell lysis was measured by ToxiLight bioluminescent cytotoxicity assay. Experiments were performed in quadruplicates with the mean \pm SEM shown for each group

was given with bendamustine (Fig. 8a). PF-06747143 strong tumor growth inhibition also resulted in significant survival benefit at the end of the study, day 75 . The hIgG1 negative control $\mathrm{Ab}$ and bendamustine groups had median survival of 27 and 40 days, respectively, while PF-06747143 monotherapy group lived significantly longer, with median survival of 68.5 days $(p<$

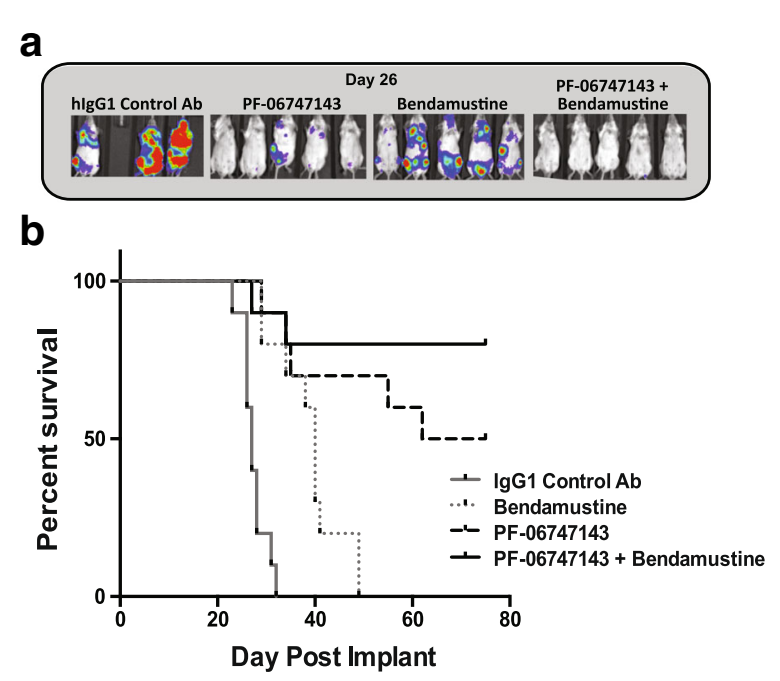

Fig. 8 PF-06747143 inhibits tumor burden and increases survival as a monotherapy or in combination with bendamustine, in a disseminated CLL tumor model. JVM-13-Luc CLL cells were implanted intravenously $\left(1 \times 10^{6}\right.$ cells $)$ and allowed to spontaneously migrate and home in the bone marrow and lymph nodes for 19 days, when animals were randomized ( $n=10 /$ group). Animals were treated with lgG1 control or PF-06747143 antibodies at $10 \mathrm{mg} / \mathrm{kg}$, subcutaneously, weekly, for six doses. Bendamustine was dosed at $30 \mathrm{mg} / \mathrm{kg}$, intraperitoneally, on days 19 and 20, followed by another 2-day cycle 28 days later a Wholebody bioluminescence representative imaging showing bone marrow tumor burden on day 26. b Kaplan-Meier survival curve, using hind leg paralysis as endpoint
0.0001 vs IgG1 control $\mathrm{Ab}$ and $p<0.0094$ vs bendamustine) (Fig. 8b). The median survival for the PF-06747143 and bendamustine combination group was not reached by the last day of the study (day 75), demonstrating a combinatorial effect between these two agents. Taken together, these results show that PF-06747143 reduces BM and lymph node tumor burden and improves survival, as a monotherapy in a CLL disseminated tumor model. Moreover, its efficacy is improved in combination with bendamustine.

\section{Discussion}

CXCR4 overexpression was shown to correlate with poor prognosis in CLL patients [23]. Activation of CXCR4 induces cell trafficking and homing of malignant cells to the BM and lymph nodes $[49,50]$, where CXCL12 is highly expressed, leading to retention of these cells in a microenvironment that provides growth signals, induces proliferation, and contributes to drug resistance, leading to poor prognosis and relapse [51]. In this study, we assessed the effect of inhibition of the CXCR4-CXCL12 pathway by a novel CXCR4 antagonist IgG1 antibody, PF-06747143, and its parental antibody m15-IgG1.

We showed that the surface expression of CXCR4 is at least tenfold higher in CLL patients than in normal $\mathrm{T}$ and B cells, in concordance with previous studies [52-54]. Similar CXCR4 expression levels were observed in highrisk or low-risk prognostic CLL patients. Importantly, both CLL risk groups had comparable sensitivity to CXCR4 antibody-induced cell death, while normal $\mathrm{T}$ and $\mathrm{B}$ cells, or the CXCR4-negative MEC-1 cell line, were largely spared. This indicates that the cell death mechanism is dependent on the level of CXCR4 surface expression. Notably, cells from CLL patients bearing the TP53mut/Del(17p) genotype, which is known to be 
resistant to F-ara-A therapy, were significantly sensitive to cell death induced by CXCR4 antibody treatment. This is of particular relevance in the treatment of refractory patients, who, in general, present with abnormal TP53 status [55]. Furthermore, the similar levels of cell death observed in CLL-B cells cultured in presence or absence of stromal cell support suggest that the CXCR4 antibody has the potential to overcome the protection provided by the microenvironment. These findings may have significant clinical implications in the treatment of patients presenting with minimal residual disease in the BM and lymph nodes.

The CXCR4 antibody significantly synergized with CLL SOC agents (rituximab, ibrutinib, F-ara-A, and bendamustine), by increasing CLL cell death. Furthermore, we demonstrated that PF-06747143 improves survival as a monotherapy and its activity is increased when combined with bendamustine in the JVM-13 mouse xenograft disseminated staged model. These findings illustrate the potential of PF-06747143 to be used in combination with these agents in the clinic.

PF-06747143 blocked CXCL12-induced cytoskeletal changes and migration of primary CLL-B cells. Although to a lesser extent, these events were also inhibited by AMD3100, the CXCR4 small molecule inhibitor. However, the lack of cell death activity shown by ADM3100 in this study, and by CXCR4 peptide inhibitors described in the literature $[4,53,56]$, suggests that just binding to CXCR4 and inhibiting CXCR4 signaling pathways is not sufficient to trigger this phenomenon. These differences may be explained by the observation that PF-06747143 induction of cell death required antibody bivalency. Bivalent binding is a property inherent to antibodies, due to their two binding regions, which are not a characteristic of small molecules or peptides. Other antiCXCR4 antibodies, including ulocuplumab, LY2624587, and hz515H7, were also shown to induce tumor cell death upon binding to CXCR4 [23, 46, 57]; however, the role of antibody bivalency in this process was not described in these studies.

In further characterizing the mechanisms involved in the cell death triggered by PF-06747143, we demonstrated that this process did not involve caspase activation. The PF-06747143 caspase-independent cell death mechanism is similar to that described for ulocuplumab, an IgG4 CXCR4 antibody [46].

Recently, antibodies binding to CD20, CD74, CD47, or HLA-DR have been shown to directly induce programmed cell death (PCD), without involvement of caspases or the need for hyper-cross-linking of the antibody [32, 58]. This novel cell death mechanism is dependent on homotypic cell adhesion triggered upon antibody binding, followed by actin redistribution and ROS activation [59,60]. Although it remains unclear which proximal events trigger PCD, the process results in loss of plasma membrane integrity and non-apoptotic cell death. A role for antibody bivalency in PCD has not been previously described; however, our results suggest that antibody bivalency might play a role in the initiating steps, by inducing homotypic cell-cell adhesion through binding to antigens expressed in adjacent cells simultaneously. We have also shown that PF-06747143 treatment generated ROS production in CLL-B cells, in association with cell death. The pattern of ROS production and cell death induced by PF-06747143 was similar to that observed with other antibodies shown to induce ROSdependent cell death, such as CD20 (obinutuzumab), TAGA1 [61], and 1D10 [62]. Taken together, our results suggest that upon binding to CXCR4 receptors in a bivalent manner, PF-06747143 triggers cell death through a caspaseindependent and bivalency-dependent mechanism, similar to PCD.

In addition to signaling blockade and induction of bivalency-dependent cell death, PF-06747143 showed potent $\mathrm{Fc}$ effector-mediated ADCC and CDC activity in CLL-B cells. Of note, PF-06747143 and m15-IgG1 ADCC activity was significantly greater than that of the m15IgG4 antibody in the ADCC assay. Similarly to m15-IgG4, ulocuplumab, which is a human IgG4 CXCR4 antibody, was recently reported to have no Fc-driven cytotoxic activity $[46,57]$. The lack of Fc effector function-driven cytotoxicity observed for the IgG4 antibodies is expected, based on the human IgG4 inherently lower affinity for the proteins involved in the process [63]. We also showed that PF-06747143 cytotoxic activity was comparable to that of obinutuzumab and rituximab [64]. The importance of ADCC or CDC activity has been clinically demonstrated for obinutuzumab and rituximab, as well as other antibodies successful therapeutic IgG1 antibodies approved for the treatment of cancers, including alemtuzumab (CD52), trastuzumab (HER2), cetuximab (EGFR), and daratumumab (CD38) $[48,65]$.

Therapeutic CXCR4 antagonists currently available lack desirable potency, cytotoxicity, safety, or adequate exposure for prolonged treatment. The small molecule AMD3100 approved for stem cell mobilization in autologous transplantation has significant safety issues that limit its chronic use [16]. In addition, we showed that AMD3100 does not induce significant cell death in B-CLL cells. Peptide antagonists of CXCR4 have been recently evaluated in clinical trials as mobilizing agents. LY2510924 was evaluated as a single agent in a phase 1 dose escalation trial in advanced metastatic cancers [66], and BKT140 (BL8040/TN14003) was evaluated in a phase 1 clinical trial in multiple myeloma $(\mathrm{MM})$ patients [19]. Treatment with both peptides induced rapid mobilization of stem cells but failed to reduce tumor burden. In addition, as for peptide therapeutics in general, they had short half-lives and required frequent administration, which makes peptides a challenging 
modality for sustained treatment [19, 66, 67]. The CXCR4 antagonist humanized IgG4 antibody, ulocuplumab, was recently evaluated in phase 1 clinical trials [20-22] and, as expected for an antibody, it exhibited a longer half-life than that of small molecules and peptides. In contrast to small molecule and peptide inhibitors of CXCR4, ulocuplumab, as well as another IgG4 CXCR4 antibody, LY26245587, and an IgG1 CXCR4 antibody hz515H7 [68], can induce tumor cell death via a mechanism reminiscent of PCD, similarly to PF06747143.

However, the IgG4 antibodies do not induce tumor cell death via ADCC or CDC $[23,46]$, as expected for human IgG4 antibodies. PF-06747143 and hz515H7 are the first IgG1 CXCR4 antibodies to be described. A key role for Fc effector functions ADCC and CDC was demonstrated when a mutation in the Fc region of hz515H7, abrogating the Fc effector function, resulted in significantly decreased efficacy in a mouse tumor model [68]. Taken together, these data suggest that the CXCR4 antibody Fc effector cytotoxic functions, ADCC and CDC, play a key role in vivo and they may contribute to efficacy enhancement in the clinic.

\section{Conclusions}

The novel CXCR4 antagonist IgG1 antibody PF06747143 binds to CXCR4 with high affinity and blocks CXCL12-induced mechanisms including calcium flux and cell migration. The CXCR4 antibody mediates CLLB cell death via a bivalency-dependent mechanism, involving generation of reactive oxygen species (ROS), with no caspase activation requirement, reminiscent of PCD. Moreover, PF-06747143 induces B-CLL cell death regardless of patient prognostic risk factor or the presence of stromal cells, indicating that it may be valuable in the treatment of resistant disease. PF-06747143 synergizes with CLL SOC agents such as bendamustine, rituximab, fludarabine, and ibrutinib. Moreover, in a CLL xenograft tumor model, PF-06747143 causes tumor growth inhibition, with increased survival, both as a monotherapy and in combination with bendamustine. Differently from other CXCR4 antagonists in the clinic, PF-06747143 induces potent cell death via Fc-driven cytotoxicity, through ADCC and CDC. Taken together, our data support the development of PF-06747143 for the treatment of CLL patients.

\section{Additional files}

Additional file 1: Figure S1. CXCR4 expression profiling in different cell lines. CXCR4 expression was performed using the 2B11 CXCR4 antibody clone for surface staining of MEC1, K562, Raji, Ramos, Jurkat, Namalwa, and JeKo-1 cell lines followed by analysis of samples using flow cytometry. The CXCR4 expression is presented as $\triangle M$ MFI. (PDF $528 \mathrm{~kb}$ )
Additional file 2: Figure S2. PF-06747143 and its Fab and F(ab')2 forms block CXCL12-induced calcium flux. The calcium flux assay was performed in human T cell leukemia Jurkat cells incubated with PF-06747143 full-length (FL), PF-06747143-Fab, PF-06747143 F(ab')2, or isotype control lgG1 antibody in presence of $\mathrm{CXCL} 12$ at $8 \mathrm{nM}$. For adequate comparison between the different forms of the antibody, their concentrations were adjusted relative to their antigen-binding site numbers. Experiment was performed in quadruplicates. The mean intracellular calcium concentration is shown in relative fluorescence units (RFU). Bars denote standard error of the mean (SEM). (PDF $389 \mathrm{~kb}$ )

Additional file 3: Figure S3. The PF-06747143 parent lgG1 antibody (m15 lgG1) induces cell death and this activity is similar in HR and LR CLL patients. Primary CLL-B cells derived from CLL patients were incubated either alone $(n=10)$ or co-cultured with stroma-NK-tert cells $(n=10)$ and treated with vehicle, IgG1 control Ab, or m15-lgG1 antibody for $48 \mathrm{~h}$. Cell death was measured using CD19/CD5/Annexin V staining followed by flow cytometry analysis. The data is derived from five high-risk (HR) and five low-risk (LR) CLL patients. The HR patients are presented with solid symbols $(\cdot)$ and LR patients denoted with hollow symbols (o). The individual data points for each group are shown. The horizontal lines represent the mean for each group Statistical comparisons were performed using Bonferroni's correction test. (PDF $744 \mathrm{~kb}$ )

Additional file 4: Figure S4. m15-lgG1 and m15-lgG4 have similar cell death activity in HR and LR CLL patients, in presence or absence of stromal cells. The primary CLL-B cells derived from CLL patients were incubated either alone $(n=4)$ or co-cultured with stroma-NK-tert cells $(n=4)$ and treated with vehicle, m15-lgG1, m15-lgG4, lgG1 control antibody, or lgG4 control antibody for 48 h. Cell death was measured using CD19/CD5/Annexin V staining followed by flow cytometry analysis. The data is presented as \% specific induced cell death (\% SICD). The data shown is derived from two high-risk (HR) and two low-risk (LR) CLL patients. The HR patients are presented with solid symbols $(\cdot)$ and LR patients denoted with hollow symbols (O). The individual data points for each group are shown. The horizontal lines represent the mean for each group. Statistical comparisons were performed using Bonferroni's correction test. (PDF 1032 kb)

Additional file 5: Figure S5. The CXCR4 antibody-induced CLL cell death increases over time, in presence or absence of stromal cells. In this washout experiment, patient CLL cells cultured alone or in presence of stroma-NK-tert cells and were treated with vehicle or m15-lgG1 antibody (200 nM) for 0.5, 2, $6,12,24,36$, and $48 \mathrm{~h}$. Cell death was measured using CD19/CD5/Annexin V staining followed by flow cytometry analysis. The samples were tested in duplicates. Statistical comparisons were performed using Bonferroni's correction test. (PDF $513 \mathrm{~kb}$ )

Additional file 6: Figure S6. m15-lgG1-induced LR or HR CLL-B cell death is independent of caspase activation. CLL-B cells were treated for $6 \mathrm{~h}$ with $\mathrm{m} 15-\lg \mathrm{g} 1(1,10$, or $100 \mathrm{nM})$ or $\lg \mathrm{G} 1$ control antibody. Caspases 3,8 , and 9 were measured using a colometric detection method. The data shown is derived from four high-risk (HR) and four low-risk (LR) CLL patients. The HR patients are denoted by triangles and LR patients denoted by circles. The individual data points for each group are shown. The horizontal lines represent the mean for each group. Statistical comparisons were performed using Bonferroni's correction test. (PDF $915 \mathrm{~kb}$ )

\section{Abbreviations}

ADCC: Antibody-dependent cell-mediated cytotoxicity; AML: Acute myeloid leukemia; CDC: Complement-dependent cytotoxicity; CLL: Chronic lymphocytic leukemia; CXCL12: C-X-C motif chemokine ligand 12; CXCR4: C-X-C chemokine receptor type 4; F-ara-A: Fludarabine; PCD: Programmed cell death; ROS: Reactive oxygen species; SICD: Specific induced cell death; SOC: Standard of care

\section{Acknowledgements}

Not applicable.

\section{Funding}

The research described herein was funded in part by Pfizer. It was also supported by the National Institutes of Health (PO1-CA081534)-CLL Research Consortium Grant to TJK and JEC, the UC San Diego Foundation Blood Cancer Research Fund to TJK, and the Bennett Family Foundation to JEC. 


\section{Availability of data and materials}

All data generated and analyzed during our study are included in this published article and its supplementary information file-Additional file 1: Figure S1.

\section{Authors' contributions}

JEC, FP, CZ, and MKK, conceived and guided the research. MKK, CA, DK, BS, $\mathrm{NH}, \mathrm{YG}, \mathrm{MH}, \mathrm{KL}$, and RY carried out the experiments. All authors analyzed and interpreted the data. JEC, MKK, and FP wrote the manuscript. SL, TS, VRF, TJK, CA, and FP critically reviewed and provided the valuable comments on the manuscript. LR, TJK, and JEC provided the patient samples and clinical data for the samples used in the study. All authors read and approved the final manuscript.

\section{Competing interests}

$B S, N H, Y G, M H, K L, R Y, M Y C, C Z, S-H L, T S$, VRF, and FP were employees of Pfizer, Inc., when the studies were conducted. The other authors declare that they have no competing interests.

\section{Consent for publication}

Not applicable.

\section{Ethics approval and consent to participate}

The local ethics committee of Pfizer as well as the UC San Diego approved the study.

\section{Author details}

${ }^{1}$ Moores Cancer Center, University of California San Diego, 3855 Health Science Drive, La Jolla, CA 92093-0820, USA. ${ }^{2}$ CLL Research Consortium, and Department of Medicine, University of California San Diego, La Jolla, CA, USA. ${ }^{3}$ Oncology Research \& Development, Pfizer Worldwide Research \& Development, 10646 Science Center Drive, San Diego, CA 92121, USA. ${ }^{4}$ Oncology Research \& Development-Rinat Biotechnology Unit, Pfizer Worldwide Research \& Development, South San Francisco, CA, USA. ${ }^{5}$ Drug Safety Research \& Development, Pfizer Worldwide Research \& Development, La Jolla, CA, USA. ${ }^{6}$ Present Address: Eli Lilly and Company, Lilly Corporate Center, Indianapolis, IN, USA. ${ }^{7}$ Present Address: ORIC Pharmaceuticals, South San Francisco, CA, USA. ${ }^{8}$ Present Address: Mirati Therapeutics, San Diego, CA, USA.

\section{Received: 15 December 2016 Accepted: 27 February 2017}

\section{Published online: 19 May 2017}

\section{References}

1. Keshava Prasad TS, Goel R, Kandasamy K, Keerthikumar S, Kumar S, Mathivanan S, Telikicherla D, Raju R, Shafreen B, Venugopal A, et al. Human Protein Reference Database—2009 update. Nucleic Acids Res. 2009;37(Database issue): D767-72.

2. Lee B, Sharron M, Montaner $L$, Weissman D, Doms RW. Quantification of CD4, CCR5, and CXCR4 levels on lymphocyte subsets, dendritic cells, and differentially conditioned monocyte-derived macrophages. Proc Natl Acad Sci U S A. 1999;96(9):5215-20.

3. Murdoch C. CXCR4: chemokine receptor extraordinaire. Immunol Rev. 2000; 177:175-84.

4. Burger M, Hartmann T, Krome M, Rawluk J, Tamamura H, Fujii N, Kipps TJ, Burger JA. Small peptide inhibitors of the CXCR4 chemokine receptor (CD184) antagonize the activation, migration, and antiapoptotic responses of CXCL12 in chronic lymphocytic leukemia B cells. Blood. 2005;106(5):1824-30.

5. Teicher BA, Fricker SP. CXCL12 (SDF-1)/CXCR4 pathway in cancer. Clin Cancer Res. 2010;16(11):2927-31.

6. Choi MY, Kashyap MK, Kumar D. The chronic lymphocytic leukemia microenvironment: beyond the B-cell receptor. Best Pract Res Clin Haematol. 2016;29(1):40-53.

7. Balkwill F. The significance of cancer cell expression of the chemokine receptor CXCR4. Semin Cancer Biol. 2004;14(3):171-9.

8. Burger JA, Bürkle A. The CXCR4 chemokine receptor in acute and chronic leukaemia: a marrow homing receptor and potential therapeutic target. Br 」 Haematol. 2007;137(4):288-96.

9. Chatterjee S, Behnam Azad B, Nimmagadda S. The intricate role of CXCR4 in cancer. Adv Cancer Res. 2014;124:31-82.
10. Guo F, Wang Y, Liu J, Mok SC, Xue F, Zhang W. CXCL12/CXCR4: a symbiotic bridge linking cancer cells and their stromal neighbors in oncogenic communication networks. Oncogene. 2016;35(7):816-26.

11. Chiorazzi N, Rai KR, Ferrarini M. Chronic lymphocytic leukemia. N Engl J Med. 2005;352(8):804-15.

12. Burger JA, Tsukada N, Burger M, Zvaifler NJ, Dell'Aquila M, Kipps TJ. Blood-derived nurse-like cells protect chronic lymphocytic leukemia B cells from spontaneous apoptosis through stromal cell-derived factor-1. Blood. 2000;96(8):2655-63.

13. Nishio M, Endo T, Tsukada N, Ohata J, Kitada S, Reed JC, Zvaifler NJ, Kipps TJ. Nurselike cells express BAFF and APRIL, which can promote survival of chronic lymphocytic leukemia cells via a paracrine pathway distinct from that of SDF-1alpha. Blood. 2005;106(3):1012-20.

14. Panayiotidis $P$, Jones D, Ganeshaguru K, Foroni L, Hoffbrand AV. Human bone marrow stromal cells prevent apoptosis and support the survival of chronic lymphocytic leukaemia cells in vitro. Br J Haematol. 1996;92(1):97-103.

15. Vianello F, Villanova F, Tisato V, Lymperi S, Ho KK, Gomes AR, Marin D, Bonnet D, Apperley J, Lam EW, et al. Bone marrow mesenchymal stromal cells nonselectively protect chronic myeloid leukemia cells from imatinib-induced apoptosis via the CXCR4/CXCL12 axis. Haematologica. 2010;95(7):1081-9.

16. DiPersio JF, Stadtmauer EA, Nademanee A, Micallef IN, Stiff PJ, Kaufman JL, Maziarz RT, Hosing C, Früehauf S, Horwitz M, et al. Plerixafor and G-CSF versus placebo and G-CSF to mobilize hematopoietic stem cells for autologous stem cell transplantation in patients with multiple myeloma. Blood. 2009;113(23):5720-6.

17. Hendrix CW, Collier AC, Lederman MM, Schols D, Pollard RB, Brown S, Jackson JB, Coombs RW, Glesby MJ, Flexner CW, et al. Safety, pharmacokinetics, and antiviral activity of AMD3100, a selective CXCR4 receptor inhibitor, in HIV-1 infection. J Acquir Immune Defic Syndr. 2004;37(2):1253-62.

18. Borthakur G NA, Ofran $Y$, Rowe JM, Altman JK, Frankfurt O, Tallman MS, Avivi I, Peled A, Pereg Y, Foley-Comer A, Russovsky L, Aharon A, McQueen T, Pemmaraju N, Bueso-Ramos CE, Cortes J, Andreeff M. BL-8040, a peptidic CXCR4 antagonist, induces leukemia cell death and specific leukemia cell mobilization in relapsed/refractory acute myeloid leukemia patients in an ongoing phase lla clinical trial. 56th Annual Meeting of the American Society of Hematology (ASH) 2014.

19. Peled A, Abraham M, Avivi I, Rowe JM, Beider K, Wald H, Tiomkin L, Ribakovsky L, Riback Y, Ramati Y, et al. The high-affinity CXCR4 antagonist BKT140 is safe and induces a robust mobilization of human CD34+ cells in patients with multiple myeloma. Clin Cancer Res. 2014;20(2):469-79.

20. Chien S, Beyerle LE, Wood BL, Estey EH, Appelbaum FR, Cardarelli PM, Sabbatini P, Shelat S, Cohen L, Becker PS. Mobilization of blasts and leukemia stem cells by anti-CXCR4 antibody BMS-936564 (MDX 1338) in patients with relapsed/refractory acute myeloid leukemia. Blood. 2013;122:3882.

21. Ghobrial I, Perez R, Baz R, Richardson P, Anderson K, Sabbatini P. Phase 1b study of the novel anti-CXCR4 antibody ulocuplumab (BMS-936564) in combination with lenalidomide plus low-dose dexamethasone, or with bortezomib plus dexamethasone in subjects with relapsed or refractory multiple myeloma. Blood. 2014;124:3483.

22. Becker PS, Foran JM, Altman JK, Yacoub A, Castro JE, Sabbatini P, et al. Targeting the CXCR4 pathway: safety, tolerability and clinical activity of ulocuplumab (BMS-936564), an anti-CXCR4 antibody, in relapsed/refractory acute myeloid leukemia. Blood. 2014;124:386.

23. Peng $S B$, Zhang $X$, Paul D, Kays LM, Ye M, Vaillancourt P, Dowless $M$, Stancato LF, Stewart J, Uhlik MT, et al. Inhibition of CXCR4 by LY2624587, a fully humanized anti-CXCR4 antibody induces apoptosis of hematologic malignancies. PLoS One. 2016;11(3):e0150585.

24. World Medical Association Declaration of Helsinki: ethical principles for medical research involving human subjects. JAMA. 2000;284(23):3043-5.

25. Tsukada N, Burger JA, Zvaifler NJ, Kipps TJ. Distinctive features of "nurselike" cells that differentiate in the context of chronic lymphocytic leukemia. Blood. 2002;99(3):1030-7.

26. Castro JE, Prada CE, Aguillon RA, Kitada S, Fukuda T, Motta M, Wu C, Dicker F, Sun G, Wang JY, et al. Thymidine-phosphorothioate oligonucleotides induce activation and apoptosis of CLL cells independently of CpG motifs or BCL-2 gene interference. Leukemia. 2006;20(4):680-8.

27. Chou TC. Drug combination studies and their synergy quantification using the Chou-Talalay method. Cancer Res. 2010;70(2):440-6.

28. Stanglmaier $\mathrm{M}$, Reis $\mathrm{S}$, Hallek $\mathrm{M}$. Rituximab and alemtuzumab induce a nonclassic, caspase-independent apoptotic pathway in B-lymphoid cell lines and in chronic lymphocytic leukemia cells. Ann Hematol. 2004; 83(10):634-45. 
29. Li HF, Wu C, Chen T, Zhang G, Zhao H, Ke CH, Xu Z. Construction and characterization of an anti-CD20 mAb nanocomb with exceptionally excellent lymphoma-suppressing activity. Int I Nanomedicine. 2015;10:4783-96.

30. Yu J, Chen L, Cui B, Widhopf GF, Shen Z, Wu R, Zhang L, Zhang S, Briggs SP, Kipps TJ. Wnt5a induces ROR1/ROR2 heterooligomerization to enhance leukemia chemotaxis and proliferation. J Clin Invest. 2016;126(2):585-98.

31. Zhang S, Wu CC, Fecteau JF, Cui B, Chen L, Zhang L, Wu R, Rassenti L, Lao F, Weigand S, et al. Targeting chronic lymphocytic leukemia cells with a humanized monoclonal antibody specific for CD44. Proc Natl Acad Sci U S A. 2013;110(15): 6127-32.

32. Honeychurch J, Alduaij W, Azizyan M, Cheadle EJ, Pelicano H, Ivanov A, Huang P, Cragg MS, Illidge TM. Antibody-induced nonapoptotic cell death in human lymphoma and leukemia cells is mediated through a novel reactive oxygen species-dependent pathway. Blood. 2012;119(15):3523-33

33. El-Daly H, Kull M, Zimmermann S, Pantic M, Waller CF, Martens UM. Selective cytotoxicity and telomere damage in leukemia cells using the telomerase inhibitor BIBR1532. Blood. 2005;105(4):1742-9.

34. Sandri S, Bobisse S, Moxley K, Lamolinara A, De Sanctis F, Boschi F, Sbarbati A, Fracasso G, Ferrarini G, Hendriks RW, et al. Feasibility of telomerasespecific adoptive T-cell therapy for B-cell chronic lymphocytic leukemia and solid malignancies. Cancer Res. 2016;76(9):2540-51.

35. Rassenti LZ, Huynh L, Toy TL, Chen L, Keating MJ, Gribben JG, Neuberg DS, Flinn IW, Rai KR, Byrd JC, et al. ZAP-70 compared with immunoglobulin heavy-chain gene mutation status as a predictor of disease progression in chronic lymphocytic leukemia. N Engl J Med. 2004;351(9):893-901.

36. Robertson LE, Chubb S, Meyn RE, Story M, Ford R, Hittelman WN, Plunkett W. Induction of apoptotic cell death in chronic lymphocytic leukemia by 2chloro-2'-deoxyadenosine and 9-beta-D-arabinosyl-2-fluoroadenine. Blood. 1993;81(1):143-50

37. Saiman Y, Jiao J, Fiel MI, Friedman SL, Aloman C, Bansal MB. Inhibition of the CXCL12/CXCR4 chemokine axis with AMD3100, a CXCR4 small molecule inhibitor, worsens murine hepatic injury. Hepatol Res. 2015; 45(7):794-803

38. Grever MR, Lucas DM, Dewald GW, Neuberg DS, Reed JC, Kitada S, Flinn IW, Tallman MS, Appelbaum FR, Larson RA, et al. Comprehensive assessment of genetic and molecular features predicting outcome in patients with chronic lymphocytic leukemia: results from the US Intergroup Phase III Trial E2997. J Clin Oncol. 2007:25(7):799-804.

39. Di Raimondo F, Palumbo GA, Romeo MA, Cacciola E, Milone G, Impera S, Giustolisi R. In vitro sensitivity of B-CLL cells to fludarabine and interferons. Leuk Lymphoma. 1995;17(5-6):449-53.

40. Rosenwald A, Chuang EY, Davis RE, Wiestner A, Alizadeh AA, Arthur DC, Mitchell JB, Marti GE, Fowler DH, Wilson WH, et al. Fludarabine treatment of patients with chronic lymphocytic leukemia induces a p53-dependent gene expression response. Blood. 2004;104(5):1428-34.

41. Ysebaert L, Gross E, Kühlein E, Blanc A, Corre J, Fournié JJ, Laurent G, QuilletMary A. Immune recovery after fludarabine-cyclophosphamide-rituximab treatment in B-chronic lymphocytic leukemia: implication for maintenance immunotherapy. Leukemia. 2010:24(7):1310-6.

42. Eichhorst B, Cramer P, Hallek M. Initial therapy of chronic lymphocytic leukemia. Semin Oncol. 2016:43(2):241-50

43. Brown JR. Ibrutinib (PCl-32765), the first BTK (Bruton's tyrosine kinase) inhibitor in clinical trials. Curr Hematol Malig Rep. 2013;8(1):1-6.

44. Bagacean C, Zdrenghea M, Tempescul A, Cristea V, Renaudineau Y. AntiCD20 monoclonal antibodies in chronic lymphocytic leukemia: from uncertainties to promises. Immunotherapy. 2016;8(5):569-81.

45. Egger L, Schneider J, Rhême C, Tapernoux M, Häcki J, Borner C. Serine proteases mediate apoptosis-like cell death and phagocytosis under caspase-inhibiting conditions. Cell Death Differ. 2003;10(10):1188-203.

46. Kashyap MK, Kumar D, Jones H, Amaya-Chanaga Cl, Choi MY, MeloCardenas J, Ale-Ali A, Kuhne MR, Sabbatini P, Cohen LJ, et al. Ulocuplumab (BMS-936564/MDX1338): a fully human anti-CXCR4 antibody induces cell death in chronic lymphocytic leukemia mediated through a reactive oxygen species-dependent pathway. Oncotarget. 2016;7(3):2809-22.

47. Burger JA, Kipps TJ. CXCR4: a key receptor in the crosstalk between tumor cells and their microenvironment. Blood. 2006;107(5):1761-7.

48. Jiang XR, Song A, Bergelson S, Arroll T, Parekh B, May K, Chung S, Strouse R, MireSluis A, Schenerman M. Advances in the assessment and control of the effector functions of therapeutic antibodies. Nat Rev Drug Discov. 2011;10(2):101-11.
49. Ten Hacken E, Burger JA. Microenvironment dependency in chronic lymphocytic leukemia: the basis for new targeted therapies. Pharmacol Ther 2014;144(3):338-48.

50. Burger JA, Montserrat E. Coming full circle: 70 years of chronic lymphocytic leukemia cell redistribution, from glucocorticoids to inhibitors of B-cell receptor signaling. Blood. 2013;121(9):1501-9.

51. Burger JA. Nurture versus nature: the microenvironment in chronic lymphocytic leukemia. Hematology Am Soc Hematol Educ Program. 2011;2011:96-103.

52. Ghobrial IM, Bone ND, Stenson MJ, Novak A, Hedin KE, Kay NE, Ansell SM. Expression of the chemokine receptors CXCR4 and CCR7 and disease progression in B-cell chronic lymphocytic leukemia/small lymphocytic lymphoma. Mayo Clin Proc. 2004;79(3):318-25.

53. Burger JA, Burger M, Kipps TJ. Chronic lymphocytic leukemia B cells express functional CXCR4 chemokine receptors that mediate spontaneous migration beneath bone marrow stromal cells. Blood. 1999:94(11):3658-67.

54. Möhle R, Failenschmid C, Bautz F, Kanz L. Overexpression of the chemokine receptor CXCR4 in B cell chronic lymphocytic leukemia is associated with increased functional response to stromal cell-derived factor-1 (SDF-1). Leukemia. 1999;13(12):1954-9.

55. Veliz M, Pinilla-Ibarz J. Treatment of relapsed or refractory chronic lymphocytic leukemia. Cancer Control. 2012;19(1):37-53.

56. Stamatopoulos B, Meuleman N, De Bruyn C, Pieters K, Mineur P, Le Roy C, Saint-Georges S, Varin-Blank N, Cymbalista F, Bron D, et al. AMD3100 disrupts the cross-talk between chronic lymphocytic leukemia cells and a mesenchymal stromal or nurse-like cell-based microenvironment: preclinical evidence for its association with chronic lymphocytic leukemia treatments. Haematologica. 2012;97(4):608-15.

57. Kuhne MR, Mulvey T, Belanger B, Chen S, Pan C, Chong C, Cao F, Niekro W, Kempe T, Henning KA, et al. BMS-936564/MDX-1338: a fully human antiCXCR4 antibody induces apoptosis in vitro and shows antitumor activity in vivo in hematologic malignancies. Clin Cancer Res. 2013;19(2):357-66.

58. Beers SA, Chan CHT, French RR, Cragg MS, Glennie MJ. CD20 as a target for therapeutic type I and II monoclonal antibodies. Semin Hematol. 2010;47(2):107-14.

59. Fleury C, Mignotte B, Vayssière JL. Mitochondrial reactive oxygen species in cell death signaling. Biochimie. 2002:84(2-3):131-41.

60. Wang $Y$, Zhu X, Yang Z, Zhao X. Honokiol induces caspase-independent paraptosis via reactive oxygen species production that is accompanied by apoptosis in leukemia cells. Biochem Biophys Res Commun. 2013;430(3):876-82.

61. Zheng JY, Tan HL, Matsudaira PT, Choo A. Excess reactive oxygen species production mediates monoclonal antibody-induced human embryonic stem cell death via oncosis. Cell Death Differ. 2017;24(3):546-58.

62. Mone AP, Huang P, Pelicano H, Cheney CM, Green JM, Tso JY, Johnson AJ, Jefferson S, Lin TS, Byrd JC. Hu1D10 induces apoptosis concurrent with activation of the AKT survival pathway in human chronic lymphocytic leukemia cells. Blood. 2004;103(5):1846-54.

63. An Z, Forrest G, Moore R, Cukan M, Haytko P, Huang L, Vitelli S, Zhao JZ, Lu $P$, Hua J, et al. lgG2m4, an engineered antibody isotype with reduced Fc function. MAbs. 2009:1(6):572-9.

64. Owen CJ, Stewart DA. Obinutuzumab for the treatment of patients with previously untreated chronic lymphocytic leukemia: overview and perspective. Ther Adv Hematol. 2015;6(4):161-70

65. Redman JM, Hill EM, AIDeghaither D, Weiner LM. Mechanisms of action of therapeutic antibodies for cancer. Mol Immunol. 2015;67(2 Pt A):28-45.

66. Galsky MD, Vogelzang NJ, Conkling P, Raddad E, Polzer J, Roberson S, Stille JR, Saleh M, Thornton D. A phase I trial of LY2510924, a CXCR4 peptide antagonist, in patients with advanced cancer. Clin Cancer Res. 2014;20(13):3581-8.

67. Oishi S, Fujii N. Peptide and peptidomimetic ligands for CXC chemokine receptor 4 (CXCR4). Org Biomol Chem. 2012;10(30):5720-31.

68. Broussas M, Boute N, Akla B, Berger S, Beau-Lavvor C, Champion T, Robert A, Beck A, Haeuw JF, Goetsch L, et al. A new anti-CXCR4 antibody that blocks the CXCR4/ SDF-1 axis and mobilizes effector cells. Mol Cancer Ther. 2016;15(8):1890-9. 